\title{
Phosphorylation of the adaptor ASC acts as a molecular switch that controls the formation of speck-like aggregates and inflammasome activity.
}

\section{$\operatorname{AUTHOR}(\mathrm{S}):$}

Hara, Hideki; Tsuchiya, Kohsuke; Kawamura, Ikuo; Fang, Rendong; Hernandez-Cuellar, Eduardo; Shen, Yanna; Mizuguchi, Junichiro; Schweighoffer, Edina; Tybulewicz, Victor; Mitsuyama, Masao

\section{CITATION:}

Hara, Hideki ... [et al]. Phosphorylation of the adaptor ASC acts as a molecular switch that controls the formation of speck-like aggregates and inflammasome activity.. Nature immunology 2013, 14(12): 1247-1255

\section{ISSUE DATE:}

2013-11-03

URL:

http://hdl.handle.net/2433/179525

\section{RIGHT:}

(C) 2013 Nature Publishing Group.; 許諾条件により本文は2014-05-04に 公開.; この論文は出版社版でありません。引用の際には出版社版をご 確認ご利用ください。; This is not the published version. Please cite only the published version. 


\title{
Phosphorylation of the adaptor ASC acts as a molecular switch that controls the formation of speck-like aggregates and inflammasome activity
}

Hideki Hara ${ }^{1,5}$, Kohsuke Tsuchiya ${ }^{1,5}$, Ikuo Kawamura1, Rendong Fang1, Eduardo Hernandez-Cuellar ${ }^{1}$, Yanna Shen ${ }^{1,2}$, Junichiro Mizuguchi ${ }^{3}$, Edina Schweighoffer ${ }^{4}$, Victor Tybulewicz $^{4} \&$ Masao Mitsuyama ${ }^{1}$

1Department of Microbiology, Kyoto University Graduate School of Medicine, Kyoto, Japan. ${ }^{2}$ School of Laboratory Medicine, Tianjin Medical University, Tianjin, China. ${ }^{3}$ Department of Immunology and Intractable Immunology Research Center, Tokyo Medical University, Tokyo, Japan. ${ }^{4}$ MRC National Institute for Medical Research, London, UK. ${ }^{5}$ H.H. and K.T. contributed equally to this work. Correspondence should be addressed to M.M. (mituyama@mb.med.kyoto-u.ac.jp).

\begin{abstract}
The inflammasome adaptor ASC contributes to innate immunity through the activation of caspase-1. Here we found that signaling pathways dependent on the kinases Syk and Jnk were required for the activation of caspase-1 via the ASC-dependent inflammasomes NLRP3 and AIM2. Inhibition of Syk or Jnk abolished the formation of ASC specks without affecting the interaction of ASC with NLRP3. ASC was phosphorylated during inflammasome activation in a Syk- and Jnk-dependent manner, which suggested that Syk and Jnk are upstream of ASC phosphorylation. Moreover, phosphorylation of Tyr144 in mouse ASC was critical for speck formation and caspase-1 activation. Our results suggest that phosphorylation of ASC controls inflammasome activity through the formation of ASC specks.
\end{abstract}


Inflammasomes are large multiprotein oligomers that serve critical roles in host defense against microbial pathogens and the development of inflammatory disorders by facilitating the secretion of proinflammatory cytokines ${ }^{1}$. Core components of each inflammasome are pro-caspase- 1 and a cytosolic pattern-recognition receptor belonging to the Nod-like receptor (NLR) family or the HIN-200 family, which contains a pyrin domain or a caspase-recruitment domain (CARD). Inflammasome complexes are believed to be assembled after the recognition of specific stimuli by the receptors ${ }^{2,3}$. Once assembled, inflammasomes serve as platforms for the activation of caspase-1, which in turn cleaves the precursor forms of interleukin-18 (IL-1B) and IL-18 to their bioactive forms ${ }^{4}$.

Different subsets of inflammasomes are activated by different stimuli. The NLRC4 inflammasome is activated by flagellin and the type III secretion apparatus from bacteria $^{5-7}$. Anthrax lethal toxin produced by Bacillus anthracistriggers activation of the NLRP1B inflammasome in mouse macrophages ${ }^{8}$. Activation of the NLRP3 inflammasome depends on a priming step (signal 1) and an activation step (signal 2) ${ }^{9}$. Signal 1 can be induced by signaling via Toll-like receptors, whereas signal 2 is induced by microbial components with diverse molecular structures, such as microbial RNA and toxins ${ }^{10,11}$. In addition, the adjuvant alum and endogenous danger-associated molecules, including ATP and monosodium urate (MSU) crystals, also induce signal 2 for the activation of the NLRP3 inflammasome ${ }^{10,12,13}$. AIM2 and IFI16 sense cytosolic DNA and nuclear DNA, respectively, and DNA viruses ${ }^{14}$, Francisella tularensis ${ }^{15}$, Listeria monocytogenes ${ }^{16,17}$ and Streptococcus pneumoniae ${ }^{18}$ have been demonstrated to activate the AIM2 inflammasome or IFI16 inflammasome ${ }^{19}$ in host cells.

The adaptor ASC (PYCARD or TMS-1) is composed of a pyrin domain and a CARD and contributes to the assembly of inflammasome complexes ${ }^{20}$. ASC serves as the bridge between pro-caspase-1 and pyrin-containing inflammasomes, such as NLRP3 and AIM2. Accordingly, NLRP3 and AIM2 require ASC exclusively for the recruitment of pro-caspase-1, while the CARD-containing receptors NLRC4 and NLRP1 can directly interact with pro-caspase-1 (refs. 1,7,21). During inflammasome activation, ASC also forms cytosolic macromolecular aggregates of ASC dimers called 'ASC specks', 'ASC foci' or 'pyroptosomes'22,23. The ASC speck recruits and activates procaspase-1, which leads to the secretion of large amounts of IL-1B and IL-18 and to pyroptosis, a form of programmed cell death. However, ASC is not always essential for cytokine processing and the induction of pyroptosis via the NLRC4 inflammasome. In addition to protein-protein interactions in inflammasome complexes, published reports have revealed the involvement of type I interferons ${ }^{15,24}$, the ubiquitin ligase-associated 
protein SGT1, the heat-shock protein hsp90 (ref. 25), unidentified serine proteases ${ }^{26}$ and kinases ${ }^{27-33}$, such as PKC- $\delta$, PKR, Syk, Lyn, PI(3)K, Erk and DAPK, in the activation or regulation of inflammasomes, but how these molecules participate in inflammasome activity remains largely unclear.

Given the importance of the protective and pathological roles of inflammasomes, it is worth clarifying what and how signaling factors are involved in the activation of inflammasomes. Here we found that the NLRP3 and AIM2 inflammasomes, but not the NLRC4 inflammasome, required Syk and Jnk for their full activity. Inhibition of Syk or Jnk abolished the NLRP3- or AIM2-mediated formation of ASC specks without affecting the interaction between ASC and NLRP3. We also found that ASC underwent Syk- and Jnk-dependent phosphorylation and that Tyr144, one of the possible phosphorylation sites, was critical for speck formation. Our results indicate the phosphorylation of ASC may be an additional target for controlling inflammasome activity. 


\section{RESULTS}

\section{NLRP3 and AIM2 require Syk and Jnk for IL-18 secretion}

To examine the role of kinases in inflammasome activation, we assessed the effects of a series of common kinase inhibitors (Supplementary Table 1) on the NLRP3, AIM2 and NLRC4 inflammasomes in peritoneal macrophages. To rule out the possibility of effects of the inhibitors on signal 1 delivered by priming with lipopolysaccharide (LPS), we added the inhibitors to macrophage cultures $3 \mathrm{~h}$ after the initiation of LPS priming. Those inhibitors did not affect the abundance of the inflammasome components or of pro-IL-18 or pro-IL-18 at the protein level (Supplementary Fig. 1a). We measured IL-18 secretion as an indicator of caspase-1 activation, as pro-IL-18, unlike pro-IL-18, is constitutively expressed in macrophages ${ }^{31}$. The nigericin-induced secretion of IL-18 from macrophages was significantly lower after pretreatment with an inhibitor of Syk or Jnk, but not after pretreatment with inhibitors of other kinases, than after pretreatment with dimethyl sulfoxide, as a control (Fig. 1a). We obtained similar results with mouse bone marrow-derived macrophages and the U937 human macrophage cell line (Supplementary Fig. 1b,c), which excluded the possibility of macrophage type- and species-specific effects. The production of IL-18 induced by alum, another NLRP3 activator, was also diminished by pretreatment with an inhibitor of Syk or Jnk (Supplementary Fig. 1e), which suggested that Syk and Jnk contributed to activation of the NLRP3 inflammasome. Moreover, we found that the production of IL-18 induced by the synthetic B-form double-stranded DNA poly(dA:dT), but not that induced by flagellin, was substantially inhibited by pretreatment with an inhibitor of Syk or Jnk (Fig. 1b,c). To confirm the role of Syk and Jnk in this, we used small interfering RNA to knock down the expression of Syk or the genes encoding Jnk1 and Jnk2 (Mapk8-Mapk9) in macrophages, and also assessed macrophages with knockout of Syk, Mapk8 or Mapk9, and found that either knockdown or knockout decreased the secretion of IL-18 in response to nigericin or poly (dA:dT) (Fig. 1d-h and Supplementary Fig. 2a-d). Also, the nigericin-induced secretion of IL-1B from macrophages was reduced by an inhibitor of Syk or Jnk or knockout of either Syk or Mapk8-Mapk9 (Fig. 1e and Supplementary Fig. 1d). Those observations suggested involvement of Syk and Jnk in activation of the NLRP3 and AIM2 inflammasomes. Syk deficiency in macrophages resulted in a moderate decrease in the secretion of IL-18 and IL-16 induced by nigericin (Fig. 1d,e), which indicated that Syk was not a critical requirement for activation of the NLRP3 inflammasome but instead contributed to that. Salmonella enterica serovar Typhimurium 14028 (S. Typhimurium) and Mycobacterium tuberculosis strain H37Rv are recognized mainly by NLRC4 and NLRP3, respectively, while Listeria 
monocytogenes strain EGD is recognized by various receptors, including AIM2 and NLRP3 (refs. 6,17,33). Consistent with the results reported above obtained by stimulation by ligands, IL-18 production induced by $S$. Typhimurium was not affected much by inhibition of Syk or Jnk in macrophages, whereas IL-18 production induced by M. tuberculosis or L. monocytogenes was reduced by inhibition of Syk or Jnk (Fig. 1i-k). From these results, we concluded that Syk and Jnk contributed to the activity of the NLRP3 and AIM2 inflammasomes but not that of the NLRC4 inflammasome.

\section{Caspase- 1 activation requires Syk and Jnk}

Next we assessed the involvement of Syk and Jnk in the activation of caspase-1 via the NLRP3 and AIM2 inflammasomes. Activation of caspase- 1 induced by nigericin, alum or poly $(\mathrm{dA}: \mathrm{dT})$ in peritoneal macrophages was almost completely abolished in the presence of an inhibitor of Syk or Jnk but not in the presence of inhibitors of other kinases (Fig. 2a,b and Supplementary Fig. 1f). In contrast, we did not observe effects of those two inhibitors on the activation of caspase-1 induced by flagellin or $S$. Typhimurium (Fig. 2c,d). Furthermore, activation of caspase-1 induced by nigericin or poly $(\mathrm{dA}: \mathrm{dT})$ was reduced in Syk- or Jnk-deficient macrophages and by knockdown of those kinases mediated by small interfering RNA (Fig. 2e-h and Supplementary Fig. $2 \mathrm{e}, \mathrm{f})$. The activation of caspase- 1 induced by $M$. tuberculosis or $L$. monocytogenes in macrophages was lower after treatment with an inhibitor Syk or Jnk than after treatment with the dimethyl sulfoxide control (data not shown). These results suggested that Syk and Jnk signals were involved in the activation of caspase-1 through the NLRP3 and AIM2 inflammasomes but not in the NLRC4 inflammasome-dependent activation of caspase-1.

\section{Syk is not required for NLRP3 inflammasome in dendritic cells}

It has been reported that Syk is not required for nigericin-induced activation of the NLRP3 inflammasome in dendritic cells ${ }^{28}$. Consistent with that report, we observed no significant difference between wild-type and Syk-deficient bone marrow-derived dendritic cells in caspase-1 activation or IL-18 secretion in response to nigericin (Supplementary Fig. 3a,c,d). In contrast, IL-18 secretion and caspase-1 activation induced by nigericin were lower in Syk-deficient peritoneal macrophages and bone marrow-derived macrophages than in $S y k^{+/+}$or $S y k^{+/-}$cells (Figs. 1d,e and 2e and Supplementary Fig. 3b,e). This suggested that the requirement for Syk in NLRP3 activation in response to nigericin was cell type specific. 


\section{Syk and Jnk regulate inflammasomes via unknown pathways}

We investigated whether inflammasome-activating agents induced activation of Syk and Jnk, assessed by the detection of phosphorylated kinases. Stimulation with nigericin or poly $(\mathrm{dA}: \mathrm{dT})$ induced detectable levels of phosphorylated Syk and Jnk (Supplementary Fig. 4a-d). The phosphorylation of Jnk induced by nigericin or poly(dA:dT) was not reduced by inhibitors of Syk or in Syk-deficient peritoneal macrophages (Supplementary Fig. 4e-g), which suggested that Syk is not upstream of Jnk in this process. Syk is reported to serve a pivotal role in activation of the NLRP3 inflammasome in response to Candida albicans by inducing the generation of reactive oxygen species (ROS) and CARD9-dependent activation of the transcription factor NF- $\mathrm{KB}^{28}$. However, we observed that wild-type and CARD9-deficient macrophages produced similar levels of IL-18 in response to nigericin or poly $(\mathrm{dA}: \mathrm{dT})$ and that the ROS scavenger BHA did not affect activation of the AIM2 inflammasome in macrophages (Supplementary Fig. 4h-j). Moreover, the expression of mitochondrial ROS, which is important for the activation of NLRP3, was not reduced by an inhibitor of Syk or Jnk in nigericin-stimulated macrophages (Supplementary Fig. 4k). These results indicated that Syk- and Jnk-dependent activation of the inflammasome is not mediated by ROS or CARD9 and that Syk and Jnk operate in a different pathway(s).

\section{The formation of ASC specks requires Syk and Jnk}

Both NLRP3 and AIM2 require the common adaptor ASC to recruit and activate pro-caspase-1 (ref. 1). We therefore speculated that Syk and Jnk might be involved in the interaction of ASC with NLRP3 or AIM2. To investigate that possibility, we visualized ASC-NLRP3 complexes by an in situ proximity ligation assay (PLA). As reported before ${ }^{30}$, we observed small spots of ASC-NLRP3 complexes in peritoneal macrophages, and these increased in abundance after stimulation with nigericin (Fig. 3a). The abundance of spots was not increased by stimulation with nigericin in the absence of ASC or NLRP3 (data not shown), which suggested that ASC-NLRP3 complexes were specifically visualized by this technique. After stimulation with nigericin, the number of spots representing ASC-NLRP3 complexes was similar in macrophages pretreated with an inhibitor of Syk or Jnk and those pretreated with dimethyl sulfoxide (Fig. 3a,b), which indicated that inhibition of Syk or Jnk did not affect the interaction between NLRP3 and ASC, a critical step in formation of the NLRP3 inflammasome, in macrophages stimulated with nigericin. Next we visualized the formation of ASC specks and found that pretreatment with an inhibitor of Syk or Jnk or deficiency in either Syk or Jnk reduced the nigericin-induced formation of ASC 
specks in macrophages (Fig. 3c-f and Supplementary Fig. 5a,b). Because ASC has been reported to form Triton X-100-resistant aggregates ${ }^{20}$, we prepared Triton X-100-soluble and Triton X-100-insoluble fractions from macrophages to analyze the distribution of ASC. ASC was almost undetectable in the Triton X-100-insoluble fraction of LPS-primed macrophages but was significantly greater in abundance after stimulation with nigericin (Fig. 3g). Moreover, most ASC in the Triton X-100-insoluble fraction was a dimer or oligomer ${ }^{15}$ (Fig. 3h). Pretreatment with an inhibitor of Syk or Jnk reduced the redistribution of ASC induced by nigericin and also resulted in a decrease in the amount of dimerized and oligomerized ASC (Fig. 3g,h). We obtained similar results for macrophages stimulated with poly(dA:dT) (Fig. 3i,j and Supplementary Fig. 5c-f). These results suggested that signaling by Syk and Jnk was required for the formation of ASC specks but not for the NLR-ASC interaction.

\section{Phosphorylation of ASC after inflammasome activation}

A published report has implied that ASC undergoes phosphorylation in response to inflammatory stimuli ${ }^{34}$. To investigate whether the aggregate formation of ASC was regulated by its phosphorylation mediated by Syk and Jnk during inflammasome activation, we enriched phosphorylated proteins from macrophage lysates through the use of a column containing a phosphorylated protein-binding resin and then detected ASC by immunoblot analysis. We observed that stimulation with nigericin induced an increase in the amount of ASC in the elution fraction (with enrichment for phosphorylated proteins; Fig. 4a). To further analyze the phosphorylation of ASC, we obtained Triton X-100-soluble and Triton X-100-insoluble cell lysates and analyzed them by a mobility-shift assay (MSA) based on the phosphate-binding tag Phos-tag ${ }^{35}$. In these gels, ASC in the Triton X-100-insoluble fraction migrated more slowly than that in the Triton X-100-soluble fraction, and the shift in mobility was reversed by phosphatase treatment (Fig. 4b,c), which suggested that ASC in the former fraction was phosphorylated. We observed the slowly migrating ASC in the Triton X-100-insoluble fraction of caspase-1-deficient macrophages but not in that of NLRP3-deficient macrophages, after stimulation with nigericin (Fig. 4b), which suggested that the increase in phosphorylated ASC in response to nigericin required NLRP3 but not caspase-1. ASC may be phosphorylated at multiple sites, as we detected three major bands with different mobility (Fig. 4c-g). In addition, deficiency in either Syk or Jnk decreased the intensity of the ASC band with the lowest mobility in the Triton $\mathrm{X}-100-$ insoluble fraction of macrophages stimulated with nigericin or poly $(\mathrm{dA}: \mathrm{dT}$ ) (Fig. $4 \mathrm{~d}-\mathrm{g}$ ). We obtained similar results with an inhibitor of Syk or Jnk (data not shown). 
These data suggested that ASC was phosphorylated after activation of the NLRP3 and AIM2 inflammasomes via the Syk and Jnk pathways. Syk and DAPK have been shown to associate with the NLRP3 inflammasome complex ${ }^{29,30}$. Accordingly, we analyzed the interaction of ASC with phosphorylated Jnk by in situ PLA. We observed complexes of ASC with phosphorylated Jnk in wild-type macrophages (Fig. 4h-k), but not in ASC-deficient macrophages (negative control; data not shown), after stimulation with nigericin or poly $(\mathrm{dA}: \mathrm{dT})$. Notably, most of the complexes were located in or around the nucleus at later time points (Fig. 4h,j). These data suggested that ASC was phosphorylated after activation of the NLRP3 and AIM2 inflammasomes via the Syk and Jnk pathways.

\section{Phosphorylation of ASC is critical for inflammasome activation}

We next sought to identify ASC-phosphorylation sites that regulate the aggregate formation that results in activation of caspase-1. We detected 14 or 8 possible phosphorylation sites, respectively, in mouse ASC with NetPhos (a neural network-based method for predicting potential phosphorylation sites at serine, threonine or tyrosine residues; version 2.0), at a threshold of 0.5, or with GPS (a group-based prediction system for the computational prediction of phosphorylation sites with their cognate kinases; version 2.1.1) with the low threshold (Supplementary Fig. 6 and Supplementary Table 2). We constructed a series of expression vectors encoding ASC mutants in which amino acid residues predicted to be phosphorylated were replaced with alanine or phenylalanine, then assessed the ability of those ASC point mutants to induce IL-1B secretion in an inflammasome-reconstitution system based on HEK293 human embryonic kidney cells (Supplementary Fig. 7a,b). We observed that the ability of the point mutants ASC(S58A), ASC(T125A), ASC(Y144F) or ASC(T151A,T152A,S153A) to induce IL-1B secretion in response to NLRP3(R258W), a disease-associated mutant of NLRP3, was slightly lower that of wild-type ASC (Fig. $5 \mathrm{a}, \mathrm{b})$. We further altered the ASC(S58A) mutant by the additional substitution T125A or with the three additional substitutions of T151A, T152A and S153A (ASC(58,151-153)) and found that IL-18-inducing ability of the resulting ASC mutants was significantly lower than that of ASC(S58A) (Fig. 5b). Furthermore, ASC(Y144F) and ASC(58,151-153) showed less redistribution into the Triton X-100-insoluble fraction in response to NLRP3(R258W) than did wild-type ASC (Fig. 5c,d). ASC(Y144F) and ASC (58,151-153) were still able to form dimers and oligomers in the Triton X-100-insoluble fraction (Fig. 5e), probably because those substitutions affected the redistribution of ASC without affecting its dimerization or oligomerization. Moreover, 
we found that ASC(Y144F) expressed together with NLRP3(R258W) formed almost no aggregates, in contrast to wild-type ASC expressed together with NLRP3(R258W) (Fig. 5f). These results suggested that Tyr144, Ser58, Thr151, Thr152 and Ser153, all putative phosphorylation sites of ASC, were involved in both IL-18-inducing ability and aggregate formation.

We investigated whether those results obtained with HEK293 cell could be reproduced in the mouse macrophage cell line RAW264.7, which lacks ASC expression. Wild-type ASC expressed together with NLRP3(R258W) in RAW264.7 cells formed speck-like structures and induced the activation of caspase-1, whereas speck formation was diminished in RAW264.7 cells transfected to express ASC(Y144F) or ASC (58,151-153) (Fig. 6a-c). The formation of ASC specks was also abrogated by the substitutions in similar experiments with ASC deficient primary macrophages (Fig. 6d).

We further investigated whether those residues were phosphorylated in a Syk- and Jnk-dependent manner. ASC expressed together with either Syk or Jnk migrated more slowly in Phos-tag gels than did ASC in cells transfected with empty vector (Fig. 7a). Transfection of cells to express ASC with both Syk and Jnk resulted in a greater mobility shift, which was reversed by phosphatase treatment (Fig. 7a,b). These results suggested that ASC was phosphorylated at multiple sites in a Syk- and Jnk-dependent manner. The observed changes in the mobility of ASC in the presence of Syk or Jnk were all abrogated by the Y144F substitution in ASC (Fig. 7c). In contrast, the mobility of ASC(S58A), ASC(T151A,T152A,S153A) and ASC(58,151-153) in Phos-tag gels, like that of wild-type ASC, was reduced by overexpression of Jnk1-Jnk2 (Fig. 7d), which suggested that Ser58, Thr151, Thr152 and Ser153 were not phosphorylated in a Jnk-dependent manner or that the effect of those substitutions on the mobility may have been masked by phosphorylation at other residues. These results showed that phosphorylation of Tyr144 was required for inflammasome activation.

We did in vitro kinase assays with synthetic peptides (amino acids 139-150 of mouse ASC or amino acids 141-152 of human ASC) to investigate the possibility that Syk directly phosphorylates Tyr144 of mouse ASC and the corresponding tyrosine residue of human ASC. We incubated those peptides with ATP in the presence or absence of recombinant Syk and assessed the kinase reaction by the consumption of ATP. We did not observe substantially less ATP in reactions in which we used those ASC peptides as a substrate for recombinant Syk, whereas reactions containing purified tubulin, as a positive control substrate, had less ATP (data not show). Thus, our data did not support or were not sufficient to justify the possibility of direct phosphorylation of Tyr144 by Syk. 


\section{MSU- and alum-induced peritonitis is dependent on Syk and Jnk}

Next we investigated whether Syk and Jnk were required for inflammasome activation in vivo. We analyzed the recruitment of inflammatory cells into the peritoneal cavity as an indicator of stimulant-induced inflammation after intraperitoneal injection of mice with MSU or alum. As shown before ${ }^{12,29}$, both MSU and alum strongly induced the recruitment of cells, including $\mathrm{Gr}^{-} 1^{+} \mathrm{F} 4 / 80^{-}$neutrophils and $\mathrm{F} 4 / 80^{+}$monocytes and macrophages, in an ASC dependent manner (Supplementary Fig. 8a-f). We then reconstituted irradiated wild-type mice with fetal liver cells to generate $S_{y k^{+/-}}$and $S y k^{-1}$ chimeras or with bone marrow cells to generate wild-type, Mapk $8^{-1-}$ and Mapk $9^{-1-}$ chimeras. After challenge with MSU or alum, $S y k^{-1-}$ chimeras had fewer total cells, neutrophils, and monocytes-macrophages in the peritoneal cavity than did $S y k^{+/-}$ chimeras (Fig. 8a,b,d-f). After challenge with MSU, the number of peritoneal monocytes-macrophages tended to be lower in $\mathrm{Syk}^{-1-}$ chimeras than in $\mathrm{Syk}^{+/-}$chimeras, but the difference between the groups was not statistically significant (Fig. 8c). The infiltration of inflammatory cells induced by MSU or alum was also lower in Mapk $8^{-1-}$ chimeras or in wild-type mice treated with an inhibitor of Jnk than in wild-type chimeras or wild-type mice treated with vehicle only, respectively (Fig. 8g-i and Supplementary Fig. 8a-f). In contrast, the inflammasome-independent infiltration of inflammatory cells induced by chemokine CXCL1 (KC) was similar in $S y k^{+/-}$and $\mathrm{Syk}^{-1}$ chimeras and in $\mathrm{Mapk}^{+/+}$and Mapk $8^{-/-}$chimeras (Supplementary Fig. 8g-1), and the inhibitor of Jnk did not reduce the infiltration of inflammatory cells induced by alum in ASC-deficient mice ( $n=3$; data not shown). These results suggested that signaling via Syk and Jnk was involved in the MSU- and alum-induced infiltration of inflammatory cells that was largely dependent on ASC. 


\section{DISCUSSION}

The phosphorylation and dephosphorylation of proteins is important in controlling a wide range of biological processes, including innate and adaptive immunity and apoptosis $^{36,37}$. The involvement of kinases such as PKC- $\delta$, PKR, Syk, Lyn, PI(3)K, Erk and DAPK in inflammasome activation has been reported, yet the precise mechanism of their action has remained unclear. In particular, Syk has been demonstrated to contribute to the NLRP3 inflammasome in response to $C$. albicans, most probably due to its role in inducing ROS production ${ }^{28,29,31,33}$. NLRC4 is phosphorylated after stimulation with its ligands, and that phosphorylation is critical for inflammasome activation ${ }^{27}$. Here we showed that Syk and Jnk were involved in the activity of ASC-containing inflammasomes in macrophages via a mechanism that regulated the formation of ASC specks. We found that ASC was phosphorylated in a Syk- and Jnk-dependent manner at multiple sites, which most probably included Tyr144, which was essential for speck formation. The phosphorylation of ASC during inflammasome activation was required for activity of the NLRP3 and AIM2 inflammasomes. Thus, inflammasomes are regulated by protein kinases and perhaps by phosphatase(s) that target phosphorylated inflammasome components.

We identified Tyr144 of ASC as a critical residue for speck formation and a possible phosphorylation site. Consistent with that, phosphorylation of the corresponding residue in human ASC (Tyr146) has been disclosed in a patent application (Hornbeck, $\mathrm{P}$. et al., US patent application 2009/0325189 A1). The Y144F substitution in ASC completely abrogated the phosphorylation of ASC induced by overexpression of Syk or Jnk, which suggested that Tyr144 serves as a regulator of ASC phosphorylation mediated by those kinases. That tyrosine residue is evolutionary conserved. Tyr144 is located in the CARD, whereas published studies have shown that the pyrin domain of ASC is phosphorylated after stimulation with tumor-necrosis factor ${ }^{34}$, which suggests the existence of other phosphorylation sites that might also contribute to inflammasome activity.

It remains unclear how signaling via Syk and Jnk regulates the formation of ASC specks. Transfection of HEK293 cells to express ASC together with Syk or Jnk or both did not result in the formation of ASC specks or the redistribution of ASC to the Triton $\mathrm{X}-100$-insoluble fraction (data not shown), which suggested that phosphorylation of ASC itself was not sufficient to induce speck formation in the absence of stimuli of the inflammasome. Our results also suggested a crucial role for Syk and Jnk in the redistribution, rather than the dimerization or oligomerization, of ASC upon inflammasome activation. During the generation of ASC aggregates, monomeric ASC, 
which is diffusely distributed before stimulation, is rapidly translocated to a perinuclear speck in each cell. The formation of ASC specks is prevented by treatment with nocodazole, and ASC specks are located near the microtubule-organizing center ${ }^{38}$, which suggests a possible role for microtubules in the migration of ASC during the step of speck formation. In our assays, most complexes of ASC and phosphorylated Jnk were located in or around the nucleus, and ASC remained diffusely distributed in the cytosol even after inflammasome activation in cells in which Syk or Jnk was inhibited genetically or pharmacologically. Thus, we speculate that Syk- and Jnk-mediated ASC phosphorylation may function as a molecular 'switch' that controls the migration of ASC along microtubules to the site of speck formation. Together, these results indicate that ASC speck formation is a consequence of multiple cellular events orchestrated by inflammasome receptors, Syk, Jnk and perhaps microtubules.

Although inflammasomes have pivotal roles in innate immunity to pathogens, excessive or dysregulated activation of inflammasomes, especially the NLRP3 inflammasome, has been linked to various autoinflammatory diseases and autoimmune diseases, including Muckle-Wells syndrome, inflammatory bowel disease $^{39,40}$, vitiligo ${ }^{41}$ and rheumatoid arthritis ${ }^{12}$. Inflammasomes have also been linked to obesity-induced insulin resistance ${ }^{42}$, atherosclerosis ${ }^{43}$ and gouty arthritis ${ }^{44}$ and could represent potential targets for therapy. Therapies involving antibody to IL-1B appear to be effective in treating inflammatory disorders associated with deregulated inflammasome activity, and further understanding of the basic processes and mechanisms involved in inflammasome activation will provide additional strategies for controlling autoinflammatory conditions. Here we have shown that phosphorylation of the inflammasome component ASC regulates inflammasome activity. Thus, for example, compounds designated to specifically inhibit the phosphorylation of ASC may be promising drug candidates for the treatment of inflammasome-associated diseases, and antibodies specific to phosphorylated ASC may be useful for diagnostic and research purposes. Further investigation of the inflammasome will reveal the precise roles of kinases in inflammasome activation and may identify additional mechanisms for controlling inflammasome activity. 


\section{ACKNOWLEDGMENTS}

We thank J. Tschopp and the Institute for Arthritis Research for permission to use Nlrp3 $^{-1-}$ mice; S. Taniguchi (Shinshu University) for Pycard ${ }^{-1}$ mice; K. Kuida (Millennium Pharmaceuticals) for Casp 1 $1^{-1-}$ mice; T. Saito (RIKEN RCAI) for Card $9^{-1-}$ mice; K. Kawasaki (Doshisha Women's College) for $S$. Typhimurium 14028; H. Tsutsui (Hyogo Medical University) for Nlrp3 $3^{-1-}$, Pycard $^{-1}$ and Casp1 $1^{-1-}$ mice; H. Hara (Saga University) for Card $9^{-/}$mice; M. Matsuura (Kyoto University) for $S$. Typhimurium 14028 and for U937 cells; H. Tanizaki (Kyoto University) for RAW264.7 cells; and K. Sada and Y. Tohyama for advice on Syk experiments. Supported by the Ministry of Education, Culture, Sports, Science and Technology of Japan, the Ministry of Health, Labour and Welfare of Japan, the Japan Society for the Promotion of Science and Medical Research Council UK (U117527252 for E.S. and V.T.). 


\section{AUTHOR CONTRIBUTIONS}

H.H. initiated the study and designed and did the experiments with macrophages and peritonitis; K.T. designed and did the experiments with HEK293 cells; K.T., H.H. and M.M. wrote the manuscript; I.K. provided advice; R.F., E.H.-C. and Y.S. contributed to the experiments; J.M. provided the Mapk $8^{-1-}$ and Mapk $9^{-1}$ mice; E.S. and V.T. provided the $S_{y k^{+/+}}, S_{y} k^{+/-}$and $S y k^{-1}$ fetal liver cells; and M.M. supervised the project. 


\section{FIGURE LEGENDS}

Figure 1 Syk and Jnk are required for IL-18 secretion mediated by NLRP3 and AIM2 but not that mediated by NLRC4. Enzyme-linked immunosorbent assay of IL-18 $(\mathrm{a}-\mathrm{d}, \mathrm{f}-\mathrm{k})$ and IL-1B (e) in peritoneal macrophages primed for $4 \mathrm{~h}$ with LPS, followed by stimulation for $90 \mathrm{~min}$ with nigericin (a,d,e,g) or for $6 \mathrm{~h}$ with encapsulated flagellin (c) or infection for $6 \mathrm{~h}$ with $S$. Typhimurium (i) or in unprimed macrophages stimulated for $3 \mathrm{~h}$ with poly(dA:dT) $(\mathrm{b}, \mathrm{f}, \mathrm{h})$ or infected for $24 \mathrm{~h}$ with $M$. tuberculosis (j) or $L$. monocytogenes $(\mathrm{k})$, without inhibitors $(\mathrm{d}-\mathrm{h})$ or with addition of the Syk inhibitors R406 $(1 \mu \mathrm{M})$, Syk inhibitor I (SI; $1 \mu \mathrm{M})$ and BAY 61-3606 (BAY; $10 \mu \mathrm{M})$, the Src inhibitor PP2 $(5 \mu \mathrm{M})$, the Jnk inhibitors SP600125 (SP; $40 \mu \mathrm{M})$ and TAT-TI-JIP153-163 (TAT; $40 \mu \mathrm{M}$ ), the p38 inhibitor SB203580 (SB; $10 \mu \mathrm{M})$, the Erk inhibitor FR180204 (FR; $10 \mu \mathrm{M})$ and the PI(3)K inhibitor wortmannin (WO; $10 \mathrm{nM}$ ) (horizontal axes) to the cultures $1 \mathrm{~h}$ before stimulation or infection for inflammasome activation (a-c,i-k). Expt, experiment. ${ }^{*} P<0.01$ and ${ }^{*} P<0.001$ (one-way analysis of variance (ANOVA) with Bonferroni's multiple-comparison test $(\mathrm{a}-\mathrm{c}, \mathrm{g}-\mathrm{k})$ or two-tailed unpaired $\mathrm{t}$-test with Welch's correction $(d-f)$ ). Data are representative of at least three independent experiments $(a-f, i-k)$ or two independent experiments ( $\mathrm{g}, \mathrm{h}$; mean and s.d. of triplicates).

Figure 2 Involvement of Syk and Jnk in NLRP3- and AIM2-mediated activation of caspase-1. Immunoblot analysis of inflammasome molecules in peritoneal macrophages primed for $4 \mathrm{~h}$ with LPS, followed by stimulation for $90 \mathrm{~min}$ with nigericin (a,e,g) or for $6 \mathrm{~h}$ with encapsulated flagellin (c) or $S$. Typhimurium (d) or in unprimed macrophages stimulated for $3 \mathrm{~h}$ with poly $(\mathrm{dA}: \mathrm{dT})(\mathrm{b}, \mathrm{f}, \mathrm{h})$, without inhibitors $(\mathrm{e}-\mathrm{h})$ or with the addition of kinase inhibitors (above lanes) to the cultures $1 \mathrm{~h}$ before stimulation (a-d). Casp1, caspase-1 (p10, subunit; p45, precursor); CL, cell lysate; SN, supernatant. Data are representative of at least three independent experiments (a-f) or two independent experiments $(\mathrm{g}, \mathrm{h})$.

Figure 3 Requirement for signaling via Syk and Jnk for ASC aggregation. (a-h) In situ PLA of ASC-NLRP3 complexes (a,b), ASC staining (c-f) and immunoblot analysis of ASC $(\mathrm{g}, \mathrm{h})$ in peritoneal macrophages primed for $4 \mathrm{~h}$ with LPS, followed by stimulation for various times (above) with nigericin in the presence or absence of kinase inhibitors. (a,c) ASC-NLRP3 complexes, red (a); ASC, green (c); nuclei, blue (a,c). Scale bars, $10 \mu \mathrm{m}$. (b) Quantification of PLA signals per cell, presented relative to that of control cells treated with solvent. (d-f) Quantification of cells with ASC specks, presented relative to that of control cells treated with solvent or wild-type cells. (g,h) Triton-soluble (S) and 
Triton-insoluble (I) fractions (right margin; g) or Triton-insoluble fractions treated with disuccinimidyl suberate (I + DSS; h). (i,j) ASC staining in unprimed macrophages stimulated for $3 \mathrm{~h}$ with poly $\left(\mathrm{dA}: \mathrm{dT}\right.$ ), presented as in $\mathrm{d}-\mathrm{f}$. ${ }^{*} P<0.001$ (Kruskal-Wallis test with Dunn's multiple-comparison test $(b, j)$, one-way ANOVA with Bonferroni's multiple-comparison test (d,f), two-tailed unpaired t-test with Welch's correction (e) or Mann-Whitney test (i)). Data are representative of at least three independent experiments $(\mathrm{a}-\mathrm{d}, \mathrm{g}, \mathrm{h})$ or two independent experiments $(\mathrm{e}, \mathrm{f}, \mathrm{i}, \mathrm{j}$; mean and s.d. of triplicates in $b, d-f, i, j)$.

Figure 4 Phosphorylation of ASC in macrophages after activation of the inflammasome. (a) Enrichment and immunoblot analysis (IB) of phosphorylated ASC in original cell lysates (Lys) and the elution (Elution) and flow-through (Flow) fractions of peritoneal macrophages primed for $4 \mathrm{~h}$ with LPS, followed by no stimulation (-) or stimulation (+) for $60 \mathrm{~min}$ with nigericin and then fractionation through a column containing a resin that binds phosphorylated proteins. $\alpha-A S C$, antibody to ASC; $\alpha-p-T y r$, antibody to phosphorylated tyrosine. (b-g) Phos-tag MSA of phosphorylated ( $\mathrm{p}^{-}$) and total ASC in Triton-soluble and Triton-insoluble fractions of peritoneal macrophages primed for $4 \mathrm{~h}$ with LPS, followed by no stimulation or stimulation for $60 \mathrm{~min}$ with nigericin (b-e), or of unprimed macrophages stimulated for $2 \mathrm{~h}$ with poly(dA:dT) (f,g), analyzed by SDS-PAGE with (+) or without (-) Phos-tag (left margin); filled triangles indicate three major bands with different mobility. Fractions were dissolved in $300 \mu \mathrm{l}$ (S) or $50 \mu \mathrm{l}$ (I) SDS sample buffer (b,d-g). (h-k) In situ PLA of peritoneal macrophages primed for $4 \mathrm{~h}$ with LPS, followed by stimulation for 0-60 min with nigericin $(\mathrm{h}, \mathrm{i})$ or of unprimed macrophages stimulated for $0-2 \mathrm{~h}$ with poly $(\mathrm{dA}: \mathrm{dT})(\mathrm{j}, \mathrm{k}) .(\mathrm{h}, \mathrm{j})$ Red, complexes of ASC with phosphorylated Jnk (ASC + p-Jnk); blue, nuclei. Scale bars, $10 \mu \mathrm{m}$. (i,k) Results presented relative to those of unstimulated control cells. ${ }^{*} P<0.01$ and ${ }^{* *} P<0.001$ (Kruskal-Wallis test with Dunn's multiple-comparison test (i) or one-way ANOVA with Tukey-Kramer multiple-comparison test $(\mathrm{k})$ ). Data are representative of two (a,c,e-g) or three $(b, d, h-k)$ independent experiments (mean and s.d. of triplicates in i,k).

Figure 5 Identification of amino acid residues in ASC critical for its biological activities. (a,b) Enzyme-linked immunosorbent assay of IL-18 in HEK293 cells $48 \mathrm{~h}$ after reconstitution by transfection of empty vector (EV) or vector encoding Flag-tagged NLRP3(R258W) (100 ng) (key) along with empty vector or vector encoding Flag-tagged wild-type (WT) or mutant ASC (10 ng; horizontal axes), plus vector encoding pro-caspase-1 (30 ng) and pro-IL-1B (100 ng). 58,125, ASC(S58A) with the additional 
substitution T125A. (c-e) Immunoblot analysis (c,e) and quantification (d) of ASC in Triton-soluble and Triton-insoluble fractions (c,d) and disuccinimidyl suberate-treated Triton-insoluble fractions (e) of reconstituted HEK293 cells $48 \mathrm{~h}$ after transfection of empty vector or vector encoding Flag-tagged NLRP3(R258W) (left margin (c), top (d) or above lanes (e)) along with vector encoding Flag-tagged wild-type or mutant ASC (left margin (c), horizontal axis (d) or above lanes (e)); in d, band intensity of insoluble ASC is presented relative to that of soluble ASC. (f) ASC staining in HEK293 cells treated as in c-e: red, ASC; green, transfected cells (visualized by transfection of a plasmid encoding green fluorescent protein $(100 \mathrm{ng}))$. Scale bars, $10 \mu \mathrm{m} .{ }^{*} P<0.05$ and ${ }^{* *} P<0.001$ (one-way ANOVA with Bonferroni's multiplecomparison test (a,b) or Tukey-Kramer's multiple-comparison test (d)). Data are from one experiment representative of three independent experiments (mean and s.d. of triplicates in a,b,d).

Figure 6 Critical roles of the possible phosphorylation sites of ASC in macrophages. (a-c) Immunoblot analysis of inflammasome molecules (a), ASC staining (b) and quantification of ASC specks (c) in reconstituted RAW264.7 cells $9 \mathrm{~h}$ after transfection of vector encoding Flag-tagged NLRP3(R258W) plus empty vector or vector encoding wild-type or mutant ASC (above lanes or images (a,b) or horizontal axis (c)). (b) ASC, green; nuclei, blue. Scale bars, $10 \mu \mathrm{m}$. (c) Results presented relative to those of the cells transfected with empty vector. (d) Quantification of ASC specks in primary Pycard ${ }^{-1}$ peritoneal macrophages $9 \mathrm{~h}$ after reconstitution by transfection of vector encoding Flag-tagged NLRP3(R258W) plus empty vector or vector encoding Flag-tagged ASC (presented as in c). ND, not detected. ${ }^{*} P<0.001$ (one-way ANOVA with Bonferroni's multiplecomparison test $(\mathrm{c}, \mathrm{d}))$. Data are from one experiment representative of three independent experiments (mean and s.d. of triplicates in c,d).

Figure 7 Identification of phosphorylation sites in ASC. (a) Phos-tag MSA of reconstituted HEK293 cells $48 \mathrm{~h}$ after transfection of vector encoding Flag-tagged wild-type ASC (50 ng) plus empty vector or vector encoding Syk (300 ng) (S) or Jnk1 (300 ng) and Jnk2 (300 ng) (J) or vectors encoding Syk, Jnk1 and Jnk2 (S+J). (b) Phos-tag MSA of reconstituted HEK293 cells $48 \mathrm{~h}$ after transfection of vector encoding Flag-tagged wild-type ASC plus empty vector or vectors encoding Syk, Jnk1 and Jnk2, followed by no treatment (-) or treatment with phosphatase (+). (c) Phostag MSA of reconstituted HEK293 cells $48 \mathrm{~h}$ after transfection of vector encoding Flag-tagged ASC(Y144F) plus vectors as in a(above lanes). (d) Phos-tag MSA of reconstituted HEK293 cells $48 \mathrm{~h}$ after transfection of vector encoding Flag-tagged wild-type or mutant 
ASC (top) plus empty vector (-) or vector Jnk1 (300 ng) and Jnk2 (300 ng) (+). Data are representative of three independent experiments.

Figure 8 Involvement of Syk and Jnk in inflammatory responses to MSU in vivo. (a-i) Absolute number of peritoneal exudate cells (PECs) (a,d,g), Gr- $1^{+} \mathrm{F} 4 / 80^{-}$neutrophils (b,e,h) and $\mathrm{F} 4 / 80^{+}$monocytes-macrophages $(\mathrm{c}, \mathrm{f}, \mathrm{i})$ in the peritoneum of chimeras (identified (horizontal axes) by cells used for reconstitution) $6 \mathrm{~h}$ after intraperitoneal injection of MSU $(a-c, g-i)$ or alum $(d-f)$. Each symbol represents an individual mouse $\left(\mathrm{n}=5\right.$ per group); small horizontal lines indicate the mean $\left( \pm\right.$ s.d.). ${ }^{*} P<0.05$ and ${ }^{* *} P<$ 0.01 (two-tailed unpaired Mann-Whitney test (a,c), two-tailed unpaired $t$-test with Welch's correction (b,d-f), one-way ANOVA with Bonferroni multiple comparison test (g) or Kruskal-Wallis test with Dunn's multiple comparison test $(\mathrm{h}, \mathrm{i})$ ). Data are representative of one experiment. 


\section{ONLINE METHODS}

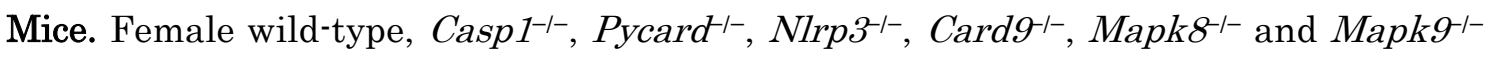
C57BL/6J mice were maintained in specific pathogen-free conditions and were used at 6-9 weeks of age ${ }^{12,16,45,46}$. C57BL/6J mice heterozygous for the $S y k^{\text {tm1Tyb }}$ allele ${ }^{47}$ were intercrossed to generate embryos at embryonic day 16.5 that were wild-type for $\operatorname{Syk}\left(S y k^{+++}\right)$or heterozygous or homozygous for the $S y k^{\text {tm1Tyb }}$ allele $\left(S y k^{+/-}\right.$or $\left.S y k^{--}\right)$. Fetal liver cells from those embryos were used to reconstitute C57BL/6J mice that had been irradiated with a total dose of $10 \mathrm{~Gy}$ from a ${ }^{137} \mathrm{Cs}$ source. Bone marrow cells from wild-type, Mapk $^{-1-}$ or Mapk9-/- mice were also used to reconstitute irradiated C57BL/6J mice. Mice were used 6-7 weeks after reconstitution. All the experimental procedures performed on mice were approved by the Animal Ethics and Research Committee of Kyoto University Graduate School of Medicine.

Cells. Peritoneal macrophages, bone marrow-derived macrophages and bone marrow-derived dendritic cells were prepared as reported ${ }^{48,49}$. Those cells suspended in culture medium consisted of RPMI-1640 medium supplemented with 10\% FCS and 10 $\mu \mathrm{g} / \mathrm{ml}$ gentamicin were incubated on culture plates overnight at $37{ }^{\circ} \mathrm{C}$, then were used for this study. U937 cells (provided by M. Matsuura) were cultured for $3 \mathrm{~d}$ in culture medium supplemented with $10 \mathrm{ng} / \mathrm{ml}$ of 12 - $O$-tetradecanoylphorbol 13 -acetate, 100 $\mathrm{U} / \mathrm{ml}$ of penicillin and $100 \mu \mathrm{g} / \mathrm{ml}$ of streptomycin. The order of treatment and positions of wells in multiwell devices were determined randomly.

Reagents. LPS and flagellin were from Invivogen; alum was from Pierce; nigericin and poly(dA: $d T)$ were from Sigma-Aldrich; MSU was from Nacalai Tesque; R406 was from Selleckchem; other kinase inhibitors were from Merck Biosciences; and BHA (butylated hydroxyanisole) was from Wako. Polyclonal antibody to ASC (AL177) and mouse monoclonal antibody (mAb) to NLRP3 (Cryo-2) were from Alexis; mAb to Jnk (56G8), mAb to phosphorylated Jnk (81E11) and mouse mAb to phosphorylated Jnk (G9) were from Cell Signaling Technology; mAb to phosphorylated Tyr (PY20), antibody to caspase-1 p10 (M-20) and antibody to Syk (anti-Syk) (N-19) were from Santa Cruz; anti-Flag (600-401-383) was from Rockland Immunochemicals; biotin-conjugated anti-IL-1B (BAF401) was from R\&D Systems; and mAb to IL-18 (39-3F) was from MBL. The enzyme-linked immunosorbent assay kit for mouse IL-1B was from eBioscience. For the 'titration' of human or mouse IL-18, a pair of biotin-labeled (93-10C) and unlabeled (74) monoclonal antibodies to IL-18 was used (both from MBL). 
Plasmids. The plasmid pmax-GFP was from Lonza. The pFLAG expression vectors for ASC, pro-caspase- 1 , and pro-IL-1B were constructed before ${ }^{16}$. The expression vectors for NLRP3 (R258W), Syk, Jnk1 (isoform B2) and Jnk2 (isoform B2) were constructed with primer sets (Supplementary Table 3) and the pFLAG-CMV2 vector (Sigma-Aldrich). After that construction, the mutation in the gene encoding NLRP3 or ASC was introduced into each expression vector by site-directed mutagenesis. Similarly, pFLAG-pro-caspase- 1 and pFLAG-pro-IL-1B in which the Flag tag was removed were generated.

Stimulation with inflammasome activators. Cells were plated at a density of $5 \times 10^{5}$ cells per well in 24-well microplates. Culture medium was replaced with Opti-MEM (Invitrogen) before stimulation or infection. Macrophages were primed for $4 \mathrm{~h}$ with 50 $\mathrm{ng} / \mathrm{ml}$ LPS and stimulated with nigericin $(5 \mu \mathrm{M})$ or alum $(500 \mu \mathrm{g} / \mathrm{ml})$. For delivery into the cytosol of macrophages, flagellin (15 ng) was encapsulated into Sendai virus envelope with GenomONE-Neo (Ishihara Sangyo). Poly(dA:dT) $(2.6 \mu \mathrm{g} / \mathrm{ml})$ was introduced into unprimed macrophages through the use of Lipofectamine LTX (Invitrogen). Cells were infected with L. monocytogenes EGD 48 (multiplicity of infection, 1) or $S$. Typhimurium 14028 (multiplicity of infection, 10), and gentamicin was added to the cultures $30 \mathrm{~min}$ after infection. Cells infected with $M$. tuberculosis H37Rv (multiplicity of infection, 5) were washed $3 \mathrm{~h}$ after infection and were further cultivated in Opti-MEM containing gentamicin. Kinase inhibitors or dimethyl sulfoxide were added to cell cultures $1 \mathrm{~h}$ before stimulation or infection for inflammasome activation.

Immunoblot analysis. Cells were lysed with SDS sample buffer. Supernatants were concentrated with trichloroacetate. For the generation of Triton X-100-soluble and Triton X-100-insoluble fractions, cells were lysed with $50 \mathrm{mM}$ Tris- $\mathrm{HCl}$ (pH 7.6) containing $0.5 \%$ Triton X-100, EDTA-free protease inhibitor 'cocktail' and phosphatase inhibitor 'cocktail' (Nacalai tesque). The lysates were centrifuged at $6,000 \mathrm{~g}$ at $4{ }^{\circ} \mathrm{C}$ for 15 min, and the pellets and supernatants were used as the Triton-insoluble and Triton-soluble fractions, respectively. For the detection of phosphorylated Syk, total Syk was immunoprecipitated with anti-Syk (N-19; Santa Cruz) and protein G Sepharose (GE Healthcare).

Crosslinkage of ASC dimers or oligomers. The Triton-insoluble pellets were washed twice with Tris-buffered saline and then resuspended in $500 \mu \mathrm{l}$ Tris-buffered saline. The resuspended pellets were crosslinked for $45 \mathrm{~min}$ at $37^{\circ} \mathrm{C}$ with $2 \mathrm{mM}$ disuccinimidyl 
suberate (Pierce) and then centrifuged for $15 \mathrm{~min}$ at 6,000 $\mathrm{g}$. The pellets were dissolved in SDS sample buffer.

Enrichment of phosphorylated proteins. Phosphorylated proteins were enriched from cell lysates with a Pro-Q Diamond Phosphoprotein Enrichment Kit according to the manufacturer's instructions (Invitrogen). Cells were lysed in lysis buffer supplemented with inhibitors of endonuclease and proteinase, then ASC aggregates were solubilized with $8 \mathrm{M}$ urea. After centrifugation for $10 \mathrm{~min}$ at $10,000 \mathrm{~g}$, the supernatants were applied to the Pro-Q diamond column ( $1 \mathrm{mg}$ protein per column). The column was washed and eluted with elution buffer (50 mM Tris, 2\% SDS, $10 \mathrm{mM}$ EDTA and 5\% 2-mercaptoethanol, $\mathrm{pH}$ 8). The flow-through and elution fractions were concentrated with trichloroacetate. ASC or phosphorylated tyrosine in each fraction was detected by immunoblot analysis.

Phos-tag-based MSA. The Triton X-100-insoluble and Triton X-100-soluble fractions from macrophages (prepared as described above) were analyzed by Phos-tag MSA. HEK293 cells were lysed with $50 \mathrm{mM}$ Tris-HCl ( $\mathrm{pH}$ 7.6) containing 1\% SDS, then lysates were precipitated with isopropanol and acetone. Proteins in the lysates were precipitated with acetone, incubated overnight at $37{ }^{\circ} \mathrm{C}$ in phosphatase reaction mixture containing $250 \mathrm{U} / \mathrm{ml}$ of antarctic phosphatase (New England BioLabs), reprecipitated with acetone and dissolved in SDS sample buffer. Those samples were separated by electrophoresis through $12 \%$ polyacrylamide gels with or without $50 \mu \mathrm{M}$ $\mathrm{MnCl}_{2}$ and $25 \mu \mathrm{M}$ Phos-tag ligand (NARD Institute).

RNA-mediated interference. Cells were transfected with $30 \mathrm{nM}$ small interfering RNA through the use of the siPORT Amine Transfection Agent (Ambion) as reported ${ }^{16}$. After $48 \mathrm{~h}$ of cultivation, the cells were washed and used in experiments. The sense small interfering RNA sequences were as follows: $\operatorname{Syk}(\mathrm{A})$, ACUUGUAGUAGUUGAUGCAUUCGGG; $\quad S y k(\mathrm{~B})$, AUUCCGAUCAUGCGCACAAUGUAGG; Mapk8(A), AAUAUAGUCCCUUCCUGGAAAGAGG; Mapk8(B), AAUUCCAGCAGAGUGAAGGUGCUUG; Mapk9(A), UAAAGUUGGUACAGGCUGUUCGCGC; and Mapkg(B), UUCAAUCGCAUGCUCUCUUUCUUCC. Stealth RNAi negative control medium GC duplex \#3 was from Invitrogen. 
Immunofluorescence staining. Cells seeded in eight-well chamber plates at a density of $2.5 \times 10^{5}$ cells per well were washed twice, fixed in $4 \%$ paraformaldehyde and permeabilized with $0.25 \%$ Triton $\mathrm{X}-100$. The cells were incubated with anti-ASC (AL177; Alexis) and then with Alexa 488-labeled or Alexa 594-labeled antibody to rabbit IgG (A11034 or A11012; Invitrogen). Nuclei were stained with DAPI (4,6-diamidino-2-phenylindole; Dojindo).

In situ PLA. Fixed and permeabilized cells were incubated overnight at $4{ }^{\circ} \mathrm{C}$ with the following pairs of primary antibodies: rabbit anti-ASC (AL177; Alexis) together with mouse mAb to phosphorylated Jnk (G9; Cell Signaling) or mouse mAb to NLRP3 (Cryo-2; Alexis). The cells were washed and allowed to react to a pair of proximity probes (Olink Bioscience). The rest of the in situ PLA protocol was performed according to the manufacturer's instructions. The cells were examined by fluorescence microscopy (Olympus), and the Duolink Image Tool (Olink Bioscience) was used for quantitative analysis.

Reconstruction of the inflammasome system in HEK293 cells. HEK293 cells (CRL-1573; American Type Culture Collection) were maintained in DMEM supplemented with 10\% FCS, $6 \mathrm{mM}$ l-glutamine, $1 \mathrm{mM}$ sodium pyruvate and $5 \mu \mathrm{g} / \mathrm{ml}$ gentamicin as described ${ }^{16}$. For experiments, HEK293 cells were plated in 24-well microplates at a density of $2 \times$ $10^{5}$ cells per well and incubated overnight. The cells were transfected with plasmids through the use of Transfectin according to the manufacturer's instructions (Bio-Rad). The total amount of DNA was adjusted to a concentration of $1 \mu \mathrm{g}$ per well with pFLAG-CMV2 empty vector. The cells were washed with culture medium $36 \mathrm{~h}$ after transfection and were further incubated for $12 \mathrm{~h}$.

Reconstruction in macrophages. RAW264.7 cells (provided by H. Tanizaki) or primary Pycard $^{-1-}$ peritoneal macrophages were transfected with 1,000 ng of pFLAG-ASC or the ASC mutant vectors together with pFLAG-NLRP3 (R258W) (250 ng) through the use of the Neon Transfection System according to the manufacturer's instructions (Invitrogen). Electroporation parameters were as follows: pulse voltage, 1,680 V; pulse width, $20 \mathrm{~ms}$; pulse number, 1 ; cell number, $1 \times 10^{6}$. The cells were incubated for $9 \mathrm{~h}$.

Peritonitis. Mice were challenged intraperitoneally with MSU (1 mg) or alum (0.4 mg). At $2 \mathrm{~h}$ before and $30 \mathrm{~min}$ after challenge with the irritants, wild-type mice were treated intraperitoneally with dimethyl sulfoxide or the Jnk inhibitor SP600125 (25 mg per kg 
body weight). The mice were killed $6 \mathrm{~h}$ after injection of the stimuli, and peritoneal cavities were lavaged with $5 \mathrm{ml}$ PBS. Mice were given intraperitoneal injection of mouse CXCL1 (0.8 $\mu \mathrm{g}$; BioLegend) or PBS, and peritoneal cells were collected $1.5 \mathrm{~h}$ after stimulation. Peritoneal cells were counted with a Countess (Invitrogen) and then were allowed to react to mAb to mouse CD16 (93; BioLegend). The cells were subsequently stained with fluorescein isothiocyanate-labeled mAb to Gr-1 (RB6-8C5; BioLegend) and phycoerythrin-labeled mAb to F4/80 (BM8; BioLegend) and were analyzed on a FACSCalibur (Becton Dickinson). Mice were prepared by H.H. and were randomly assigned to experimental groups. Another investigator (R.F.), who did the injections and flow cytometry, was 'blinded' to the identity of the groups.

Statistical analysis. The sample size of in vivo and in vitro studies was chosen to be as small as possible but to allow the evaluation of distribution normality. For two-group comparisons by Gaussian distribution, a two-tailed unpaired $t$ test with Welch's correction was used when the variances of the groups were judged to be equal by the $F$ test. For two-group comparisons with non-Gaussian distribution, a Mann-Whitney test was used. Multigroup comparisons with Gaussian distribution, one-way ANOVA with Bonferroni's multiple-comparison test or Tukey-Kramer's multiple-comparison test (for samples of unequal size) was used after the confirmation of homogeneity of variance among the groups by Bartlett's test. For multigroup comparisons with non-Gaussian distribution, a Kruskal-Wallis test with Dunn's test was used. $P$ values of 0.05 or less were the threshold for statistical significance. 


\section{REFERENCES}

1. Martinon, F., Mayor, A. \& Tschopp, J. The inflammasomes: guardians of the body. Annu. Rev. Immunol. 27, 229-265 (2009).

2. Brodsky, I.E. \& Monack, D. NLR-mediated control of inflammasome assembly in the host response against bacterial pathogens. Semin. Immunol. 21, 199-207 (2009).

3. Davis, B.K., Wen, H. \& Ting, J.P. The inflammasome NLRs in immunity, inflammation, and associated diseases. Annu. Rev. Immunol. 29, 707-735 (2011).

4. Dinarello, C.A. Immunological and inflammatory functions of the interleukin-1 family. Annu. Rev. Immunol. 27, 519-550 (2009).

5. Miao, E.A. et al. Innate immune detection of the type III secretion apparatus through the NLRC4 inflammasome. Proc. Natl. Acad. Sci. USA 107, 3076-3080 (2010).

6. Franchi, L. et al. Cytosolic flagellin requires Ipaf for activation of caspase-1 and interleukin 18 in salmonella-infected macrophages. Nat. Immunol. 7, 576-582 (2006).

7. Miao, E.A. et al. Cytoplasmic flagellin activates caspase-1 and secretion of interleukin 1beta via Ipaf. Nat. Immunol. 7, 569-575 (2006).

8. Boyden, E.D. \& Dietrich, W.F. Nalp1b controls mouse macrophage susceptibility to anthrax lethal toxin. Nat. Genet. 38, 240-244 (2006).

9. Franchi, L., Munoz-Planillo, R., Reimer, T., Eigenbrod, T. \& Nunez, G. Inflammasomes as microbial sensors. Eur. J. Immunol. 40, 611-615 (2010).

10. Mariathasan, S. et al. Cryopyrin activates the inflammasome in response to toxins and ATP. Nature 440, 228-232 (2006).

11. Kanneganti, T.D. et al. Critical role for Cryopyrin/Nalp3 in activation of caspase-1 in response to viral infection and double-stranded RNA. J. Biol. Chem. 281, 36560-36568 (2006).

12. Martinon, F., Petrilli, V., Mayor, A., Tardivel, A. \& Tschopp, J. Gout-associated uric acid crystals activate the NALP3 inflammasome. Nature 440, 237-241 (2006).

13. Li, H., Nookala, S. \& Re, F. Aluminum hydroxide adjuvants activate caspase-1 and induce IL-1beta and IL-18 release. J. Immunol. 178, 5271-5276 (2007).

14. Rathinam, V.A. et al. The AIM2 inflammasome is essential for host defense against cytosolic bacteria and DNA viruses. Nat. Immunol. 11, 395-402 (2010).

15. Fernandes-Alnemri, T. et al. The AIM2 inflammasome is critical for innate immunity to Francisella tularensis. Nat. Immunol. 11, 385-393 (2010).

16. Tsuchiya, K. et al. Involvement of absent in melanoma 2 in inflammasome activation in macrophages infected with Listeria monocytogenes. J. Immunol. 185, 1186-1195 (2010).

17. Kim, S. et al. Listeria monocytogenes is sensed by the NLRP3 and AIM2 
inflammasome. Eur. J. Immunol. 40, 1545-1551 (2010).

18. Fang, R. et al. Critical roles of ASC inflammasomes in caspase-1 activation and host innate resistance to Streptococcus pneumoniae infection. J. Immunol. 187, 4890-4899 (2011).

19. Kerur, N. et al. IFI16 acts as a nuclear pathogen sensor to induce the inflammasome in response to Kaposi sarcoma-associated herpesvirus infection. Cell Host Microbe 9, 363-375 (2011).

20. Masumoto, J. et al. ASC, a novel $22-\mathrm{kDa}$ protein, aggregates during apoptosis of human promyelocytic leukemia HL-60 cells. J. Biol. Chem. 274, 33835-33838 (1999).

21. Case, C.L., Shin, S. \& Roy, C.R. Asc and Ipaf Inflammasomes direct distinct pathways for caspase-1 activation in response to Legionella pneumophila. Infect. Immun. 77, 1981-1991 (2009).

22. Bryan, N.B., Dorfleutner, A., Rojanasakul, Y. \& Stehlik, C. Activation of inflammasomes requires intracellular redistribution of the apoptotic speck-like protein containing a caspase recruitment domain. J. Immunol. 182, 3173-3182 (2009).

23. Fernandes-Alnemri, T. et al. The pyroptosome: a supramolecular assembly of ASC dimers mediating inflammatory cell death via caspase-1 activation. Cell Death Differ. 14, 1590-1604 (2007).

24. Guarda, G. et al. Type I interferon inhibits interleukin-1 production and inflammasome activation. Immunity 34, 213-223 (2011).

25. Mayor, A., Martinon, F., De Smedt, T., Petrilli, V. \& Tschopp, J. A crucial function of SGT1 and HSP90 in inflammasome activity links mammalian and plant innate immune responses. Nat. Immunol. 8, 497-503 (2007).

26. Franchi, L. et al. Calcium-independent phospholipase A2B is dispensable in inflammasome activation and its inhibition by bromoenol lactone. J. Innate Immun. 1, 607-617 (2009).

27. Qu, Y. et al. Phosphorylation of NLRC4 is critical for inflammasome activation. Nature 490, 539-542 (2012).

28. Gross, O. et al. Syk kinase signalling couples to the Nlrp3 inflammasome for antifungal host defence. Nature 459, 433-436 (2009).

29. Shio, M.T. et al. Malarial hemozoin activates the NLRP3 inflammasome through Lyn and Syk kinases. PLoS Pathog. 5, e1000559 (2009).

30. Chuang, Y.T. et al. Tumor suppressor death-associated protein kinase is required for full IL-1B production. Blood 117, 960-970 (2011).

31. Kankkunen, P. et al. (1,3)-beta-glucans activate both dectin-1 and NLRP3 inflammasome in human macrophages. J. Immunol. 184, 6335-6342 (2010). 
32. Lu, B. et al. Novel role of PKR in inflammasome activation and HMGB1 release. Nature 488, 670-674 (2012).

33. Wong, K.W. \& Jacobs, W.R. Jr. Critical role for NLRP3 in necrotic death triggered by Mycobacterium tuberculosis. Cell Microbiol. 13, 1371-1384 (2011).

34. Stehlik, C. et al. The PAAD/PYRIN-only protein POP1/ASC2 is a modulator of ASC mediated nuclear-factor- $\mathrm{kB}$ and pro-caspase-1 regulation. Biochem. J. 373, 101-113 (2003).

35. Kosako, H. et al. Phosphoproteomics reveals new ERK MAP kinase targets and links ERK to nucleoporin-mediated nuclear transport. Nat. Struct. Mol. Biol. 16, 1026-1035 (2009).

36. Kurokawa, M. \& Kornbluth, S. Caspases and kinases in a death grip. Cell 138, 838-854 (2009).

37. Sumbayev, V.V. \& Yasinska, I.M. Role of MAP kinase-dependent apoptotic pathway in innate immune responses and viral infection. Scand. J. Immunol. 63, 391-400 (2006). 38. Balci-Peynircioglu, B. et al. Expression of ASC in renal tissues of familial mediterranean fever patients with amyloidosis: postulating a role for ASC in AA type amyloid deposition. Exp. Biol. Med. (Maywood) 233, 1324-1333 (2008).

39. Villani, A.C. et al. Common variants in the NLRP3 region contribute to Crohn's disease susceptibility. Nat. Genet. 41, 71-76 (2009).

40. Zaki, M.H. et al. The NLRP3 inflammasome protects against loss of epithelial integrity and mortality during experimental colitis. Immunity 32, 379-391 (2010).

41. Jin, Y. et al. NALP1 in vitiligo-associated multiple autoimmune disease. N. Engl. J. Med. 356, 1216-1225 (2007).

42. Vandanmagsar, B. et al. The NLRP3 inflammasome instigates obesity-induced inflammation and insulin resistance. Nat. Med. 17, 179-188 (2011).

43. Duewell, P. et al. NLRP3 inflammasomes are required for atherogenesis and activated by cholesterol crystals. Nature 464, 1357-1361 (2010).

44. Pope, R.M. \& Tschopp, J. The role of interleukin-1 and the inflammasome in gout: implications for therapy. Arthritis Rheum. 56, 3183-3188 (2007).

45. Hara, H. et al. The adaptor protein CARD9 is essential for the activation of myeloid cells through ITAM-associated and Toll-like receptors. Nat. Immunol. 8, 619-629 (2007). 46. Cao, Y. et al. Enhanced T cell-independent antibody responses in c-Jun N-terminal kinase 2 (JNK2)-deficient B cells following stimulation with CpG-1826 and anti-IgM. Immunol. Lett. 132, 38-44 (2010).

47. Turner, M. et al. Perinatal lethality and blocked B-cell development in mice lacking the tyrosine kinase Syk. Nature 378, 298-302 (1995). 
48. Hara, H. et al. Dependency of caspase-1 activation induced in macrophages by Listeria monocytogenes on cytolysin, listeriolysin $\mathrm{O}$, after evasion from phagosome into the cytoplasm. J. Immunol. 180, 7859-7868 (2008).

49. Lutz, M.B. et al. An advanced culture method for generating large quantities of highly pure dendritic cells from mouse bone marrow. J. Immunol. Methods 223, 77-92 (1999). 

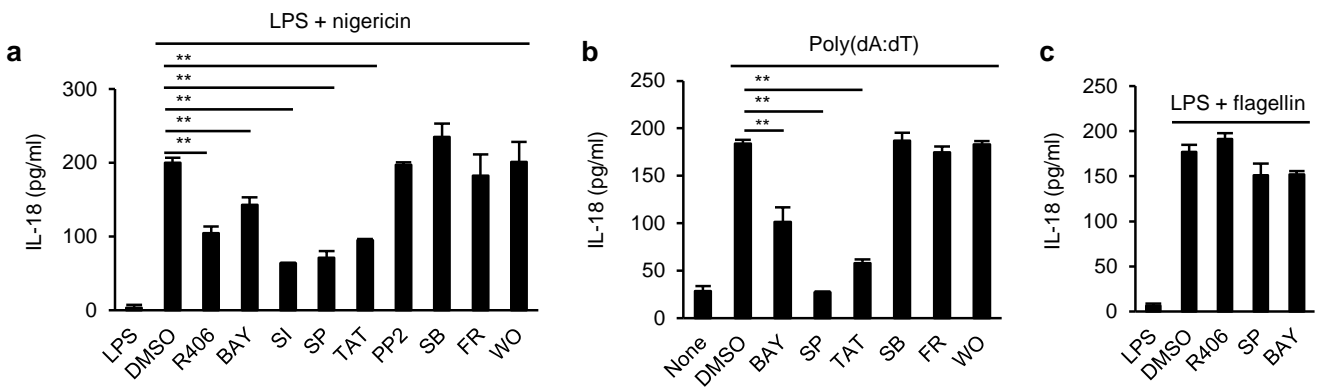

d

Expt 2

Expt 3
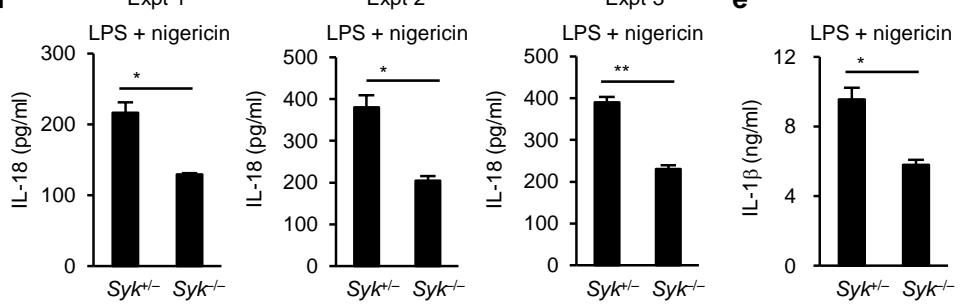

f

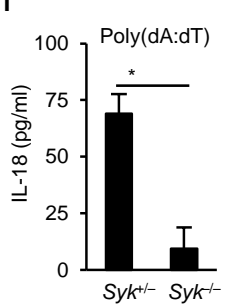

g
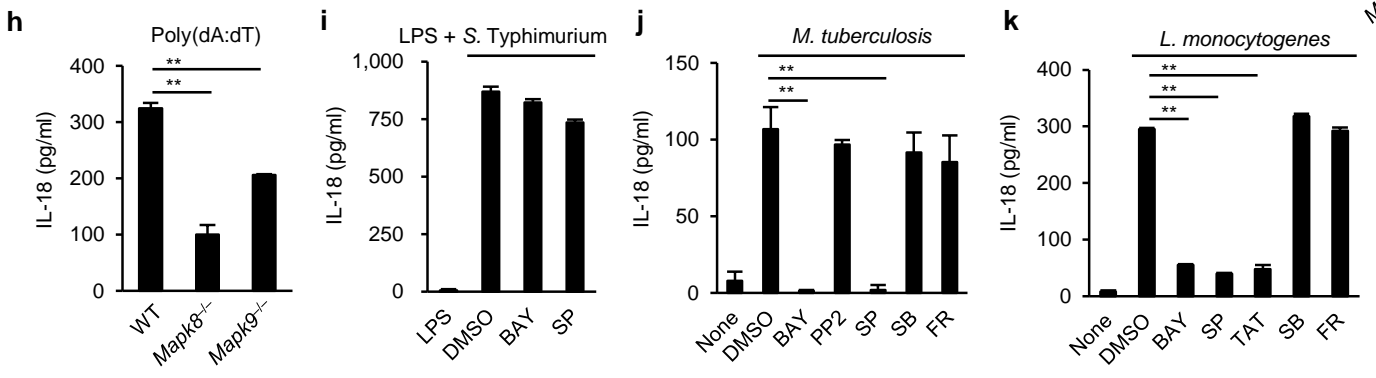

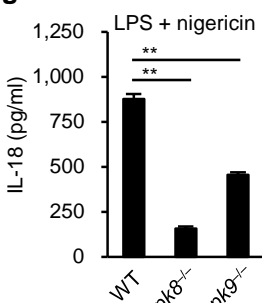


a

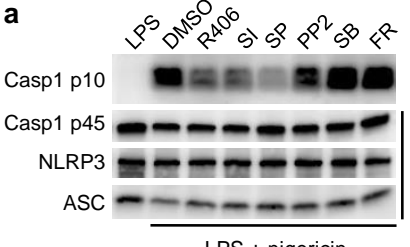

LPS + nigericin

b

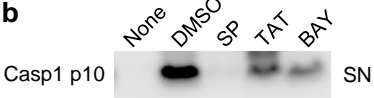

Casp1 p45 - - - -

ASC

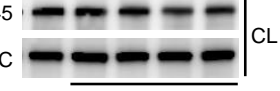

Poly(dA:dT)

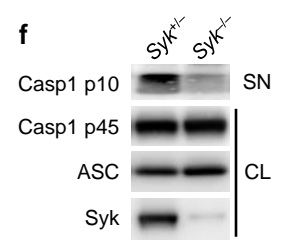

Poly(dA:dT) c

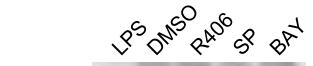

SN Casp1p10 $\mathrm{SN}$

Casp1 p45 - - $-\mathrm{CL}$ $\mathrm{CL}$

d

$\overline{\text { LPS + flagellin }}$

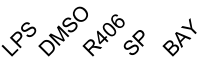

Casp1 p10 OND

Casp1 p45 $-\infty \mathrm{CL}$

LPS + S. Typhimurium

Expt 1

s) 5$)^{x^{x^{x}}}$

Casp1 p10 $=\mathrm{SN}$

Casp1 p45

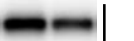

NLRP3 - CL

ASC

Syk

LPS + nigericin
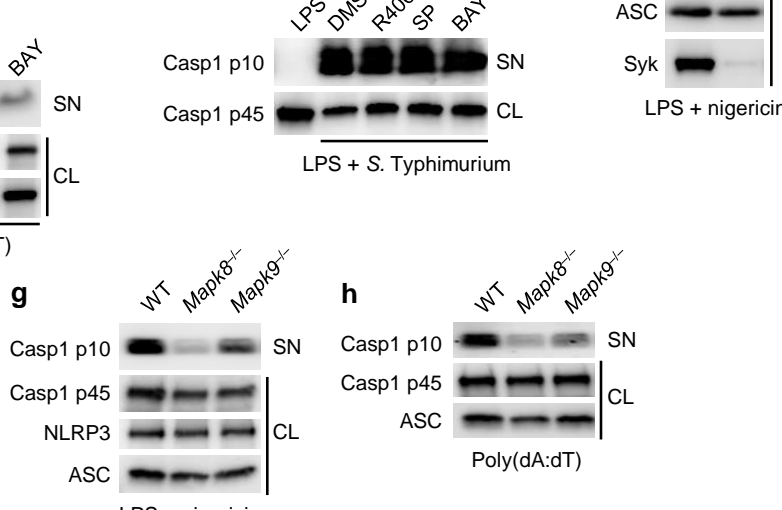

h

Casp1 p10

Casp1 p45

ASC

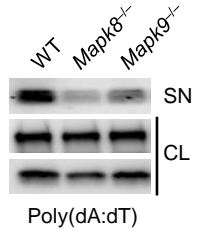

Expt 2

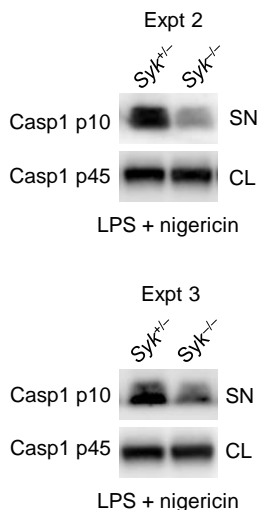

LPS + nigericin

LPS + nigericin 
a

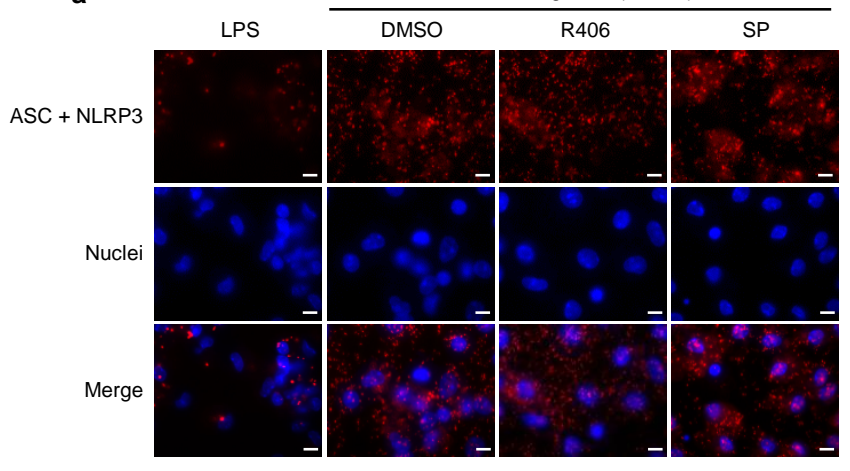

C

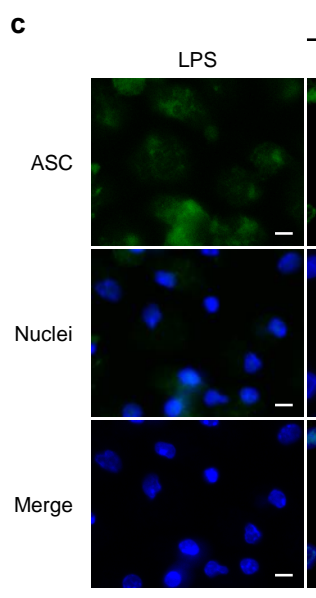

h

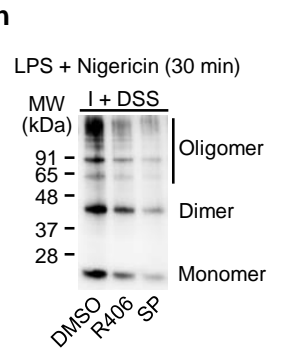

LPS + nigericin (90 min)
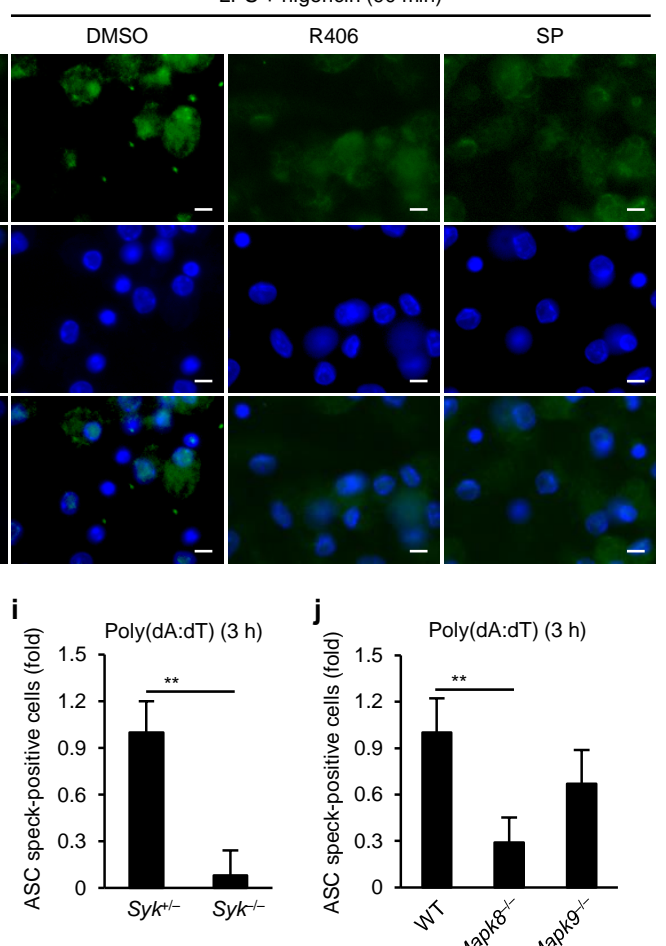

b

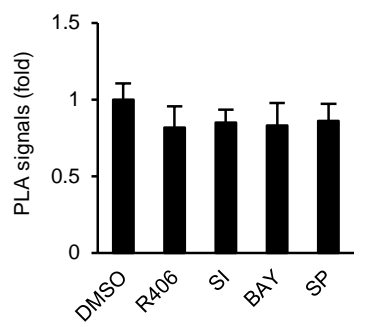

e

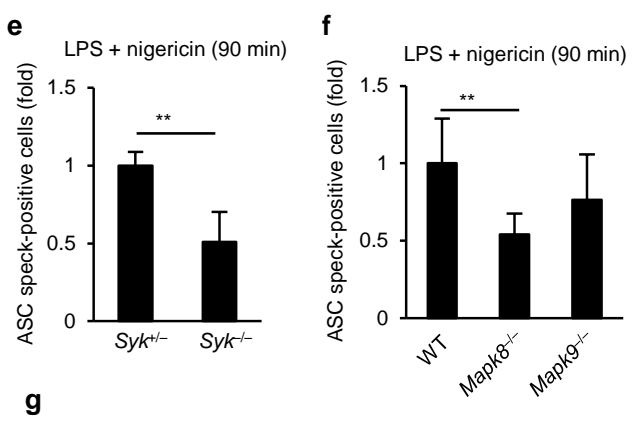

LPS +
nigericin $\frac{\text { DMSO }}{03060} \frac{\mathrm{R} 406}{03060} \frac{\mathrm{SP}}{03060 \text { (min) }}$

ASC

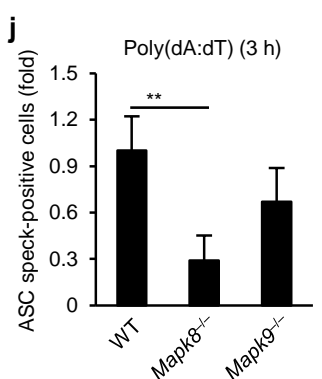

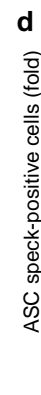
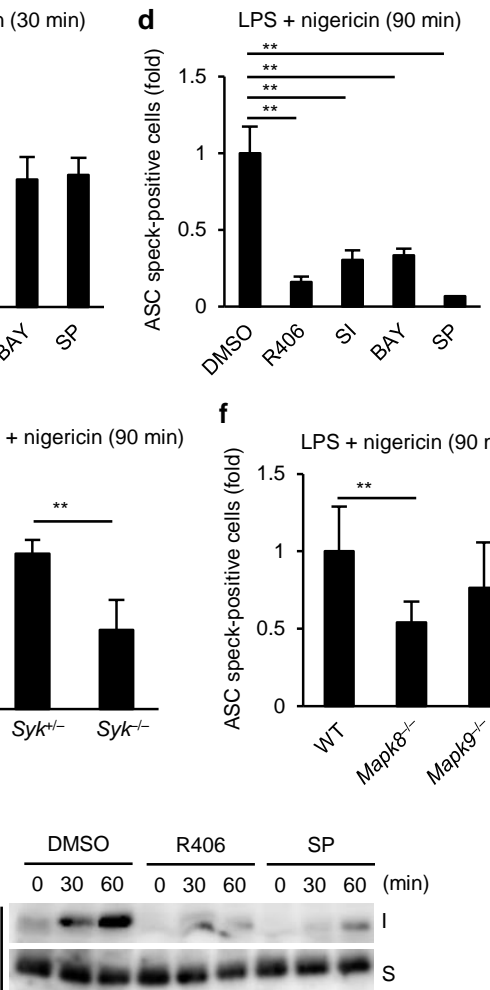
a

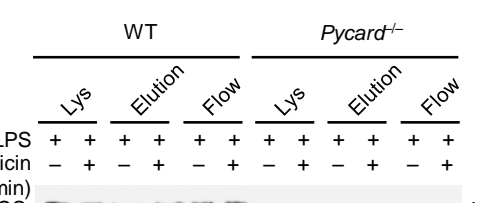
Nigericin
(60 min) ASC

250

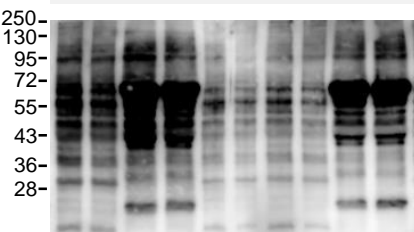

17-

(kDa)

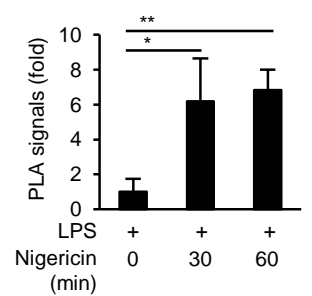

k

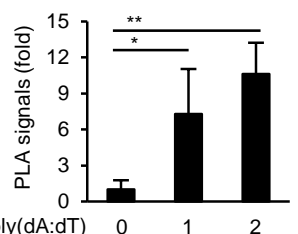

(h)

h

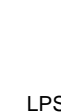

LPS
+ nigericin
$(60 \mathrm{~min})$

+ nigericin (30 min)

+ nigericin$$
\text { (1) }
$$

b

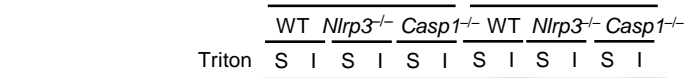

IB: $\alpha$-ASC

Phos-tag $(+$

Phos-tag (-)

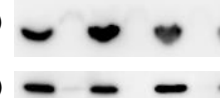

d

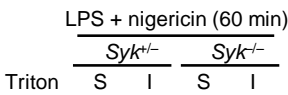

IB: $\alpha-p-T y r$

Phos-tag (+)

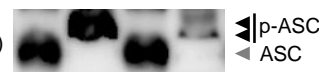

f

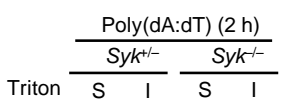

Phos-tag $(+)$
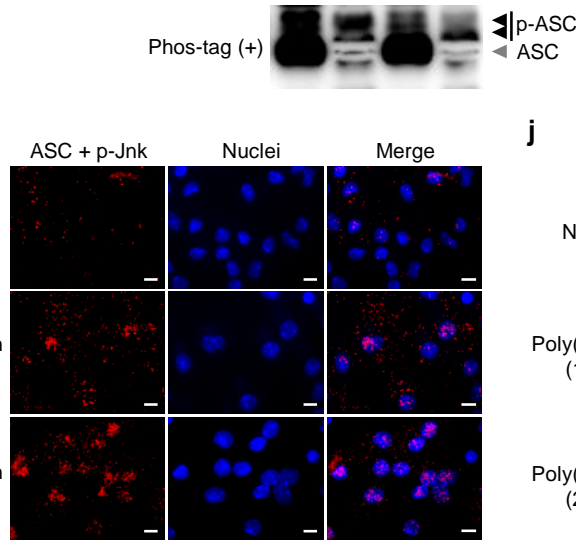

Nuclei

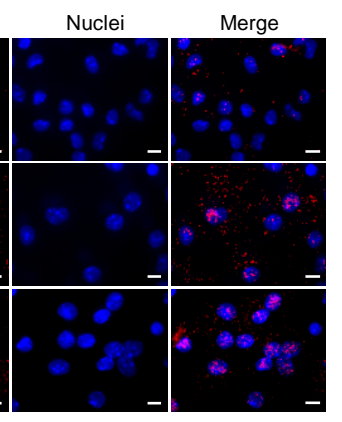

c

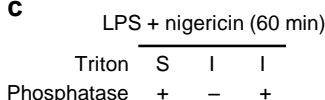

Phos-tag (+)

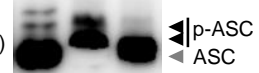

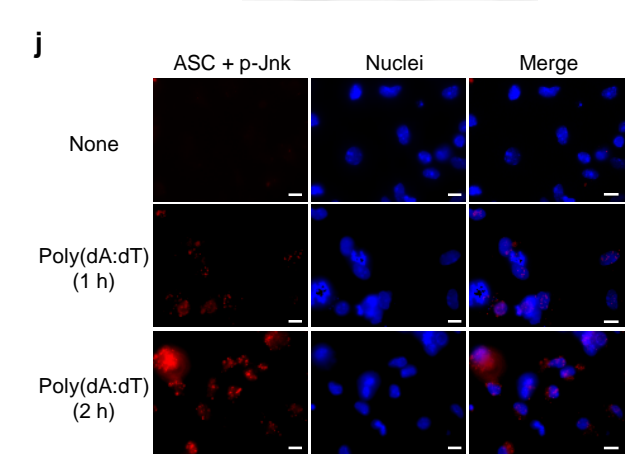

e

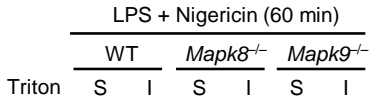

Phos-tag (+)

g

Triton $\frac{\text { Poly }(\mathrm{dA}: \mathrm{dT})(2 \mathrm{~h})}{\mathrm{WT}} \frac{M a p k 8^{-1-}}{\mathrm{S} \text { I }} \frac{M a p k 9^{-1-}}{\mathrm{S} \text { I }}$

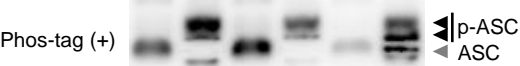

ASC 
a

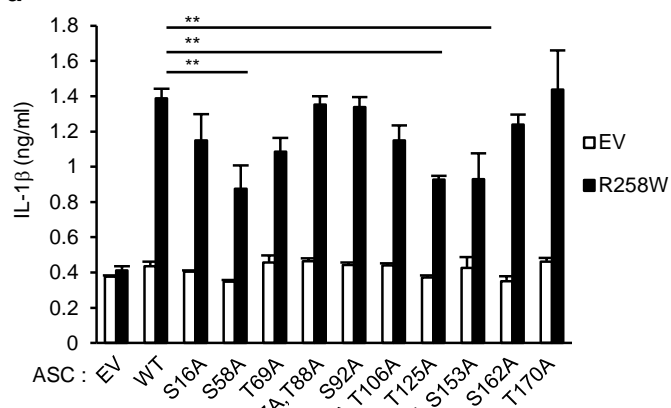

b

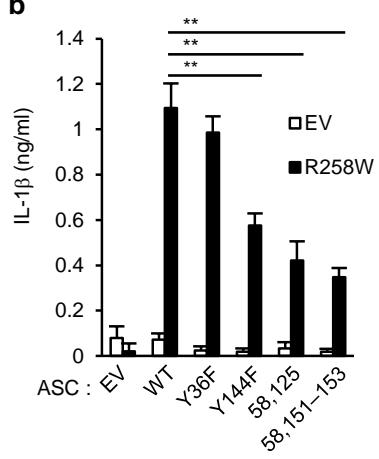

C

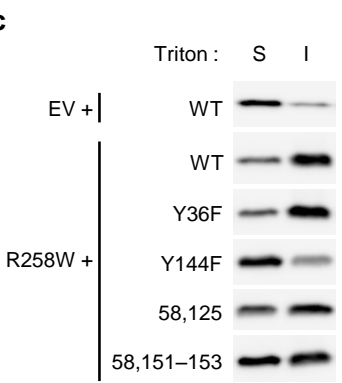

IB: $\alpha$-FLAG
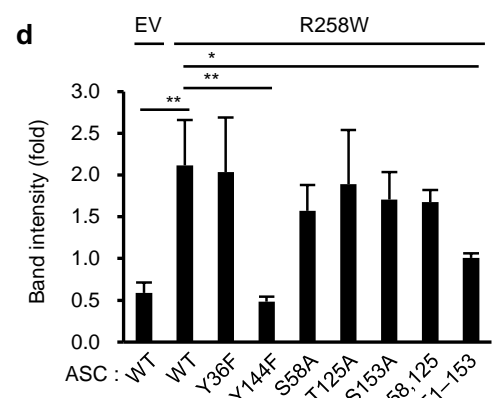

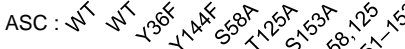
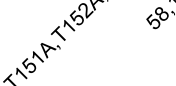

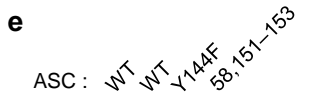

R258W

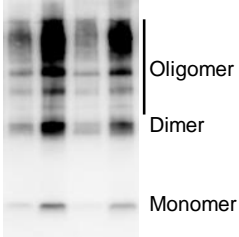

IB: $\alpha$-ASC

f

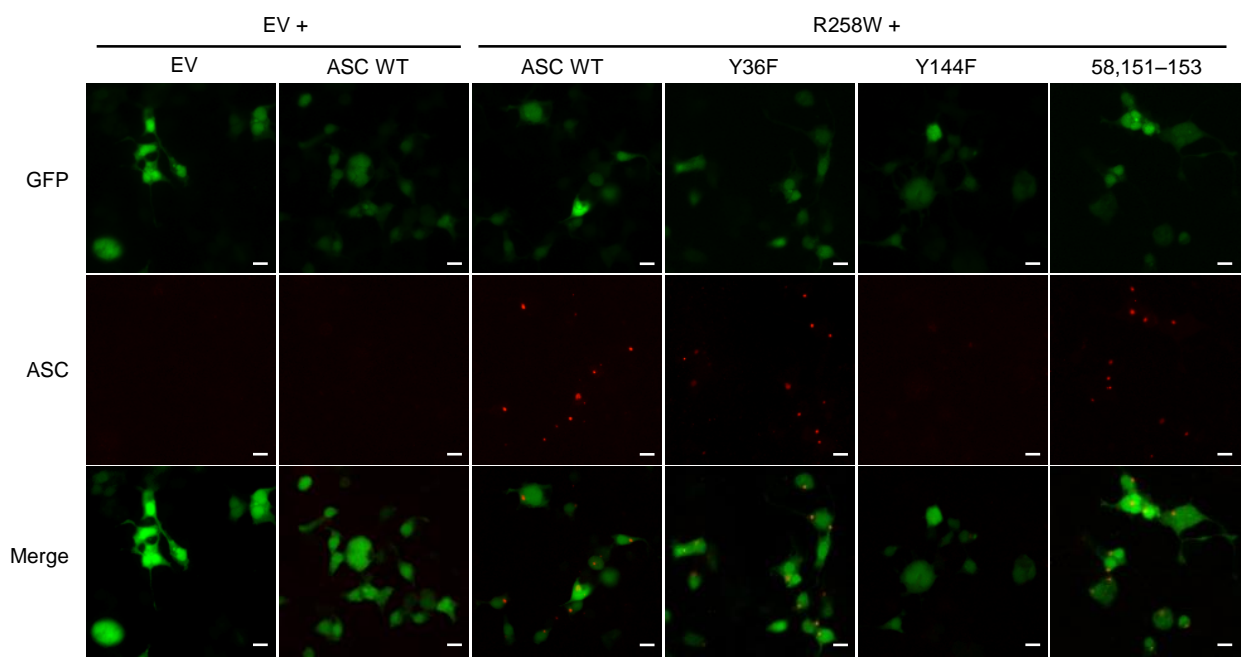



a

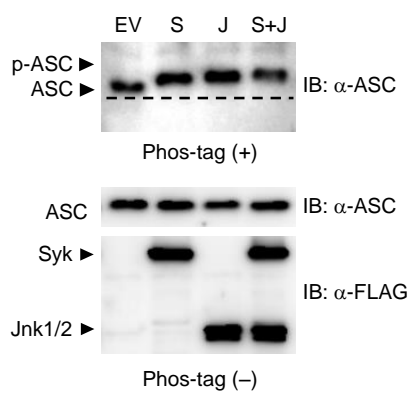

b

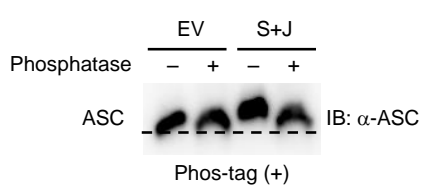

c

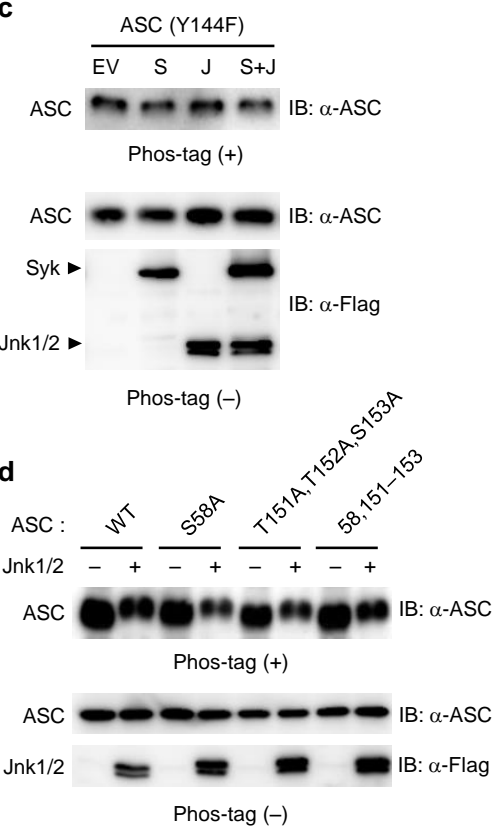

ASC

ASC 
京都大学

Kyoto University Research Information Repository

KURENAI 红

a

b

C

d

e
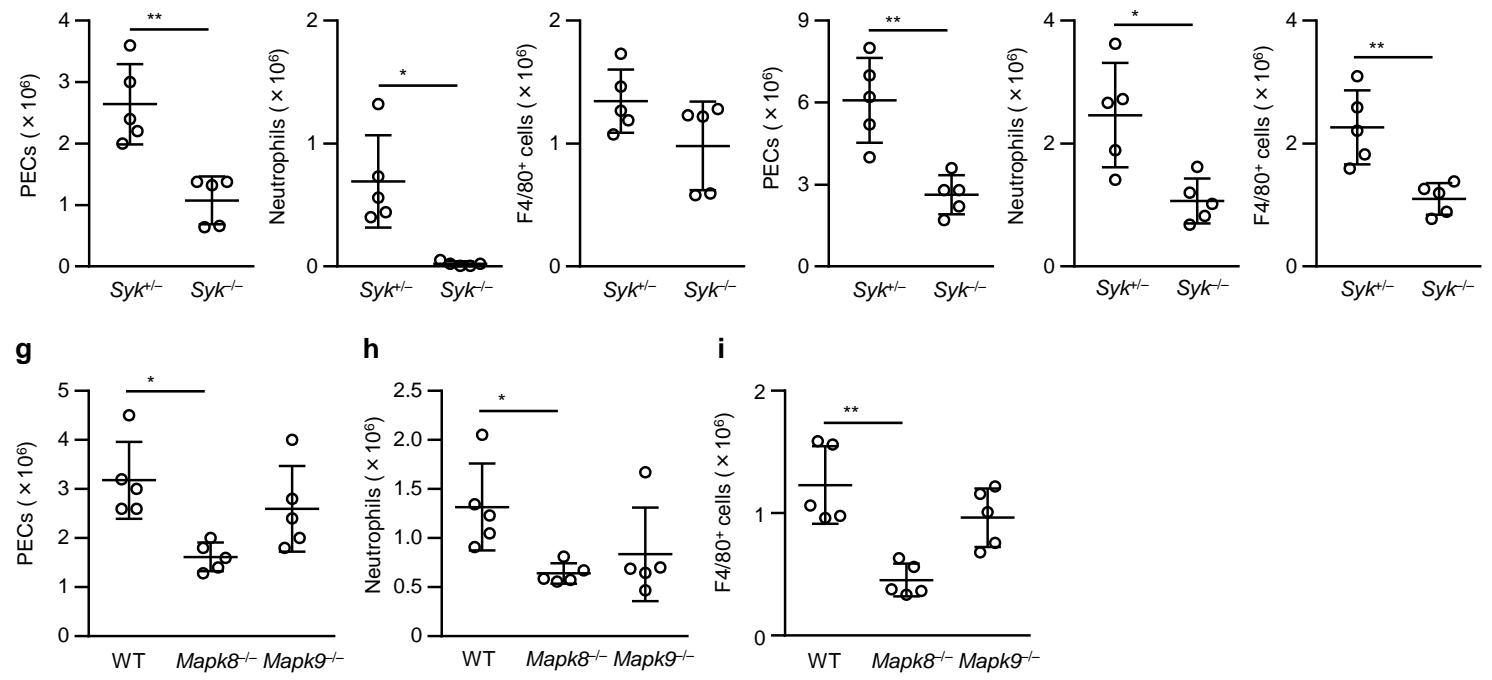

h
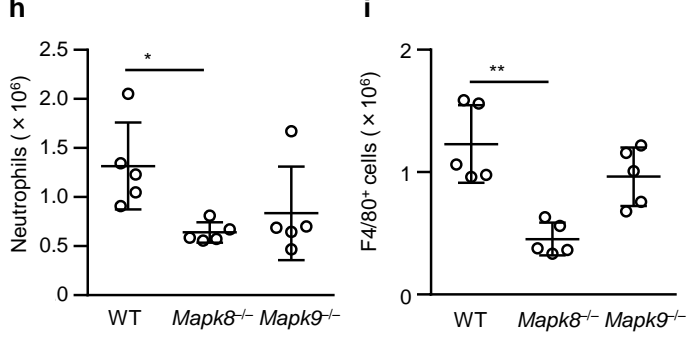
a

$\frac{\text { Non-stimulation }}{\text { - DMSO R406 BAY SI SP TAT }}$

$$
\text { LPS }
$$

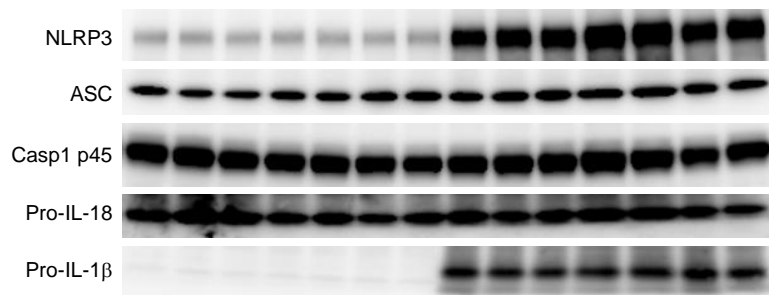

b

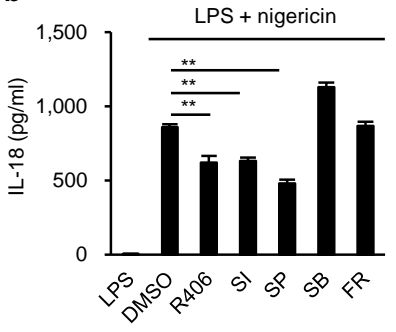

C

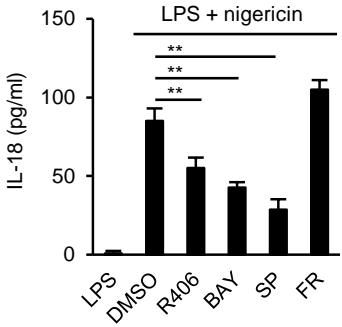

d

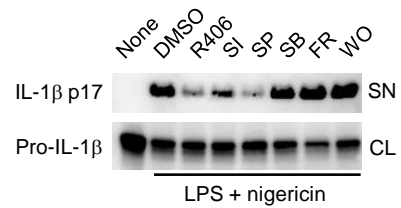

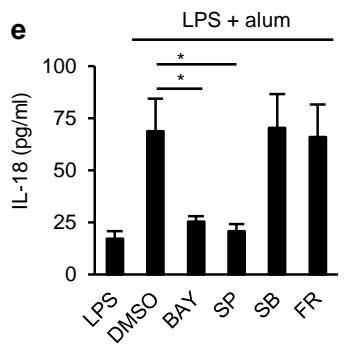

f

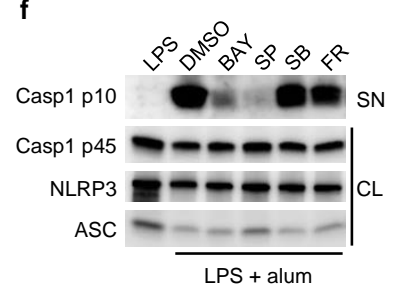

Supplementary Figure 1 Inhibition of Syk or Jnk do not affect the expression of inflammasome molecules. (a,d,f) Immunoblot analysis of inflammasome molecules or (b,c,e) Enzyme-linked immunosorbent assay of IL-18 in peritoneal macrophages (a,d-f), bone marrow-derived macrophages (b), or U937 cells (c) primed with LPS for 4 $\mathrm{h}$ (a), followed by stimulation with nigericin for $90 \mathrm{~min}(\mathbf{b}-\mathbf{d})$, or alum for $6 \mathrm{~h}(\mathbf{e}, \mathbf{f})$. The indicated kinase inhibitors were added to the cultures $1 \mathrm{~h}$ before stimulation. CL, cell lysates; SN, supernatants. Data are shown as the means \pm s.d. of triplicate samples of one experiment representative of three independent experiments. Data were analyzed by one-way ANOVA with Bonferroni multiple comparison test $(\mathbf{b}, \mathbf{c}, \mathbf{e}) .{ }^{*} P<0.01$ and ${ }^{* *} P<0.001$. 
a

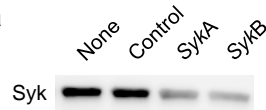

b Jnk

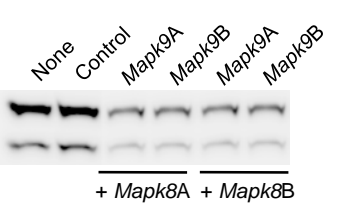

C हิ

a

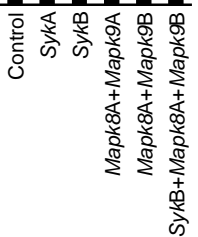

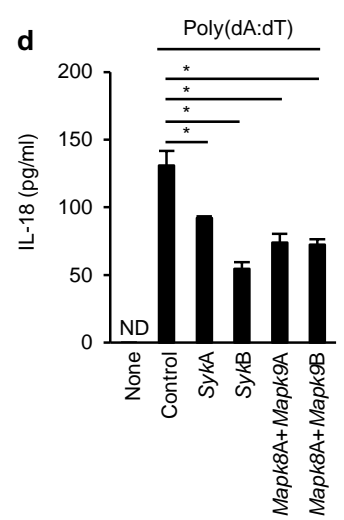

LPS + nigericin

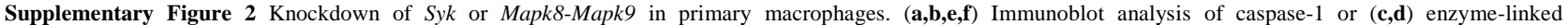

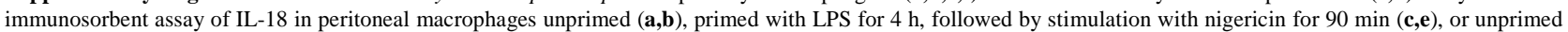

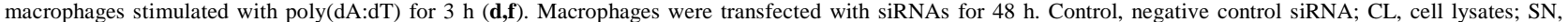

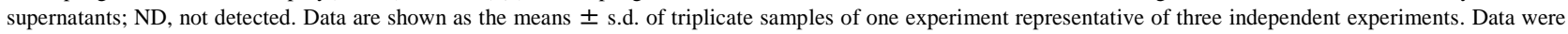
analyzed by one-way ANOVA with Bonferroni multiple comparison test (c,d). ${ }^{*} P<0.001$. 
a

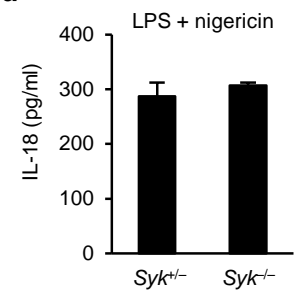

b

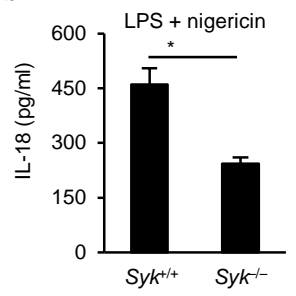

c

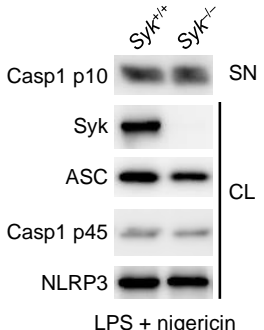

d

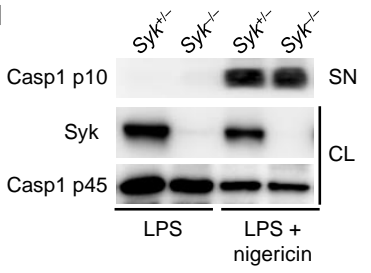

e

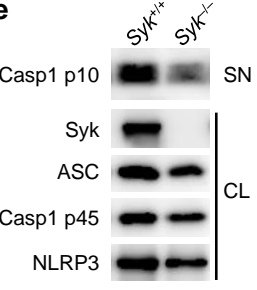

LPS + nigericin

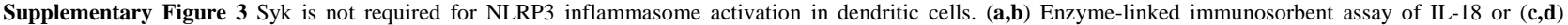

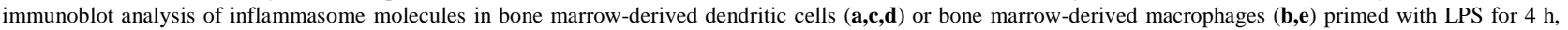

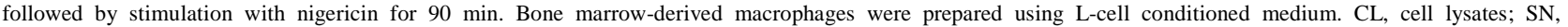

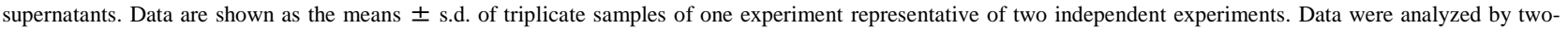
tailed unpaired $t$ test with Welch's correction (a,b). ${ }^{*} P<0.01$ 


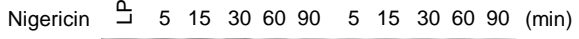

p-Syk $--m-m-m$ IP: $\alpha$-Syk

Syk

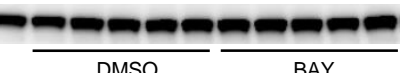

B: $\alpha-p-T y r$

DMSO BAY

b

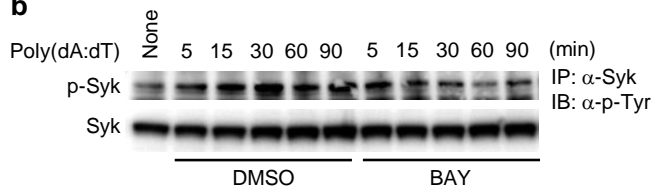

C

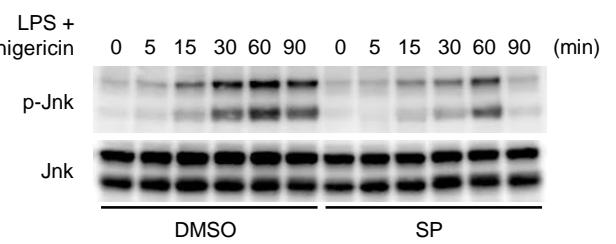

d

$\begin{array}{llllllllllllll}\text { Poly(dA:dT) } & 0 & 5 & 15 & 30 & 60 & 90 & 0 & 5 & 15 & 30 & 60 & 90 & \text { (min) }\end{array}$

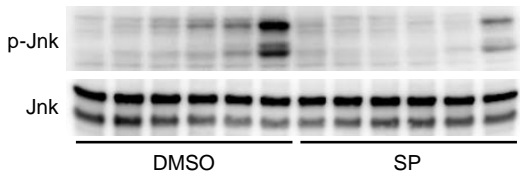

LPS +

nigericin $\quad 0 \quad 30 \quad 60 \quad 90 \quad 0 \quad 30 \quad 60 \quad 90 \quad(m i n)$

p-Jnk

Jnk

f

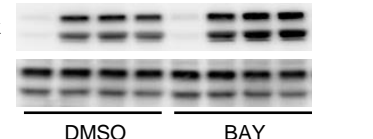

Poly(dA:dT) $0 \quad 30 \quad 60 \quad 90 \quad 0 \quad 30 \quad 60 \quad 90$ (min)

p-Jnk

Jnk

g

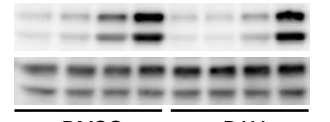

LPS +

DMSO $=\frac{-1-T}{\text { BAY }}$

$\begin{array}{llllllll}\text { nigericin } & 0 & 30 & 60 & 0 & 30 & 60 & (\mathrm{~min})\end{array}$

p-Jnk

Jnk

$\frac{------}{S y k^{+-}} \frac{-}{S y k^{\prime-}}$

h

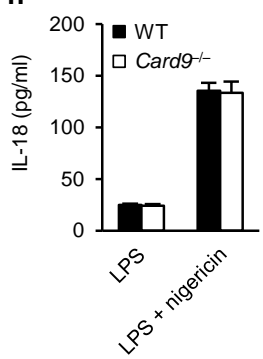

$\mathbf{k}$

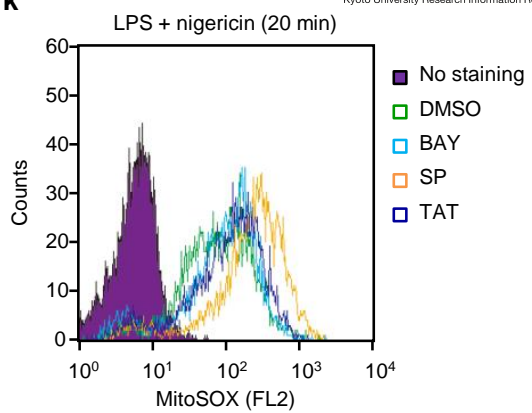

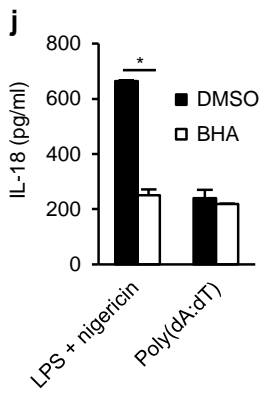

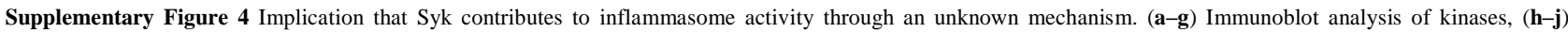

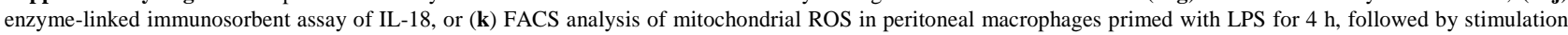

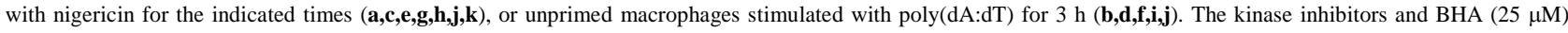

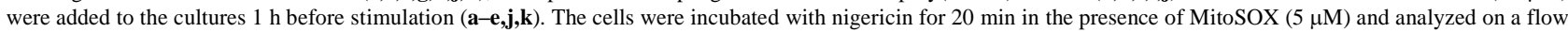

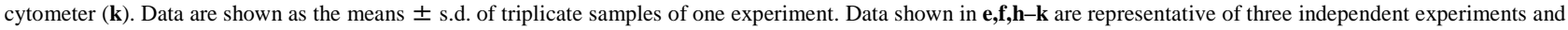
those in $\mathbf{a}-\mathbf{d}, \mathbf{g}$ are representative of two independent experiments. Data were analyzed by two-tailed unpaired $t$ test with Welch's correction $(\mathbf{h}-\mathbf{j})$. ${ }^{*} P<0.001$. 
京都大学

KYOTO UNIVERSITY

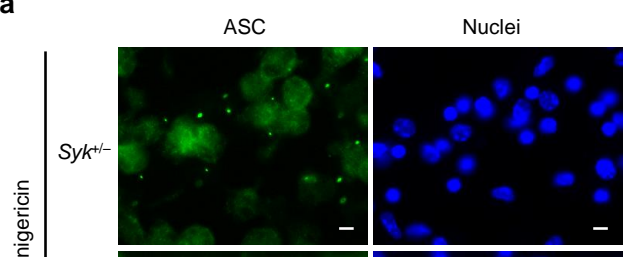

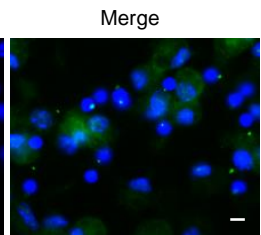

b

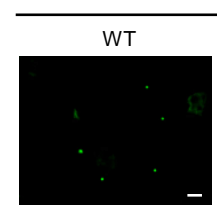

Mapk8-1- $^{-1}$
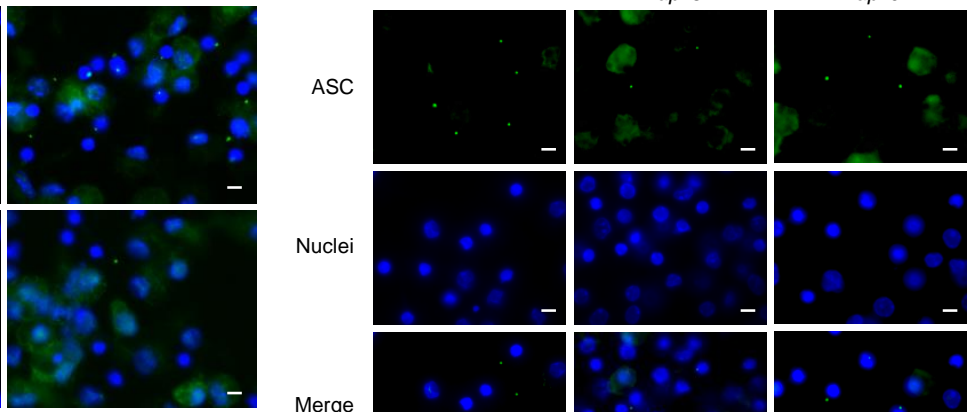

Merge
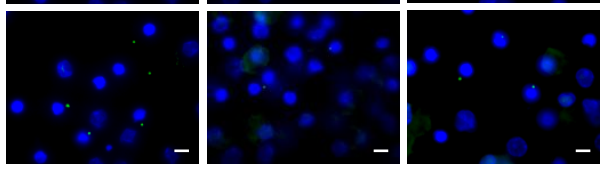

Poly(dA:dT) (3 h)

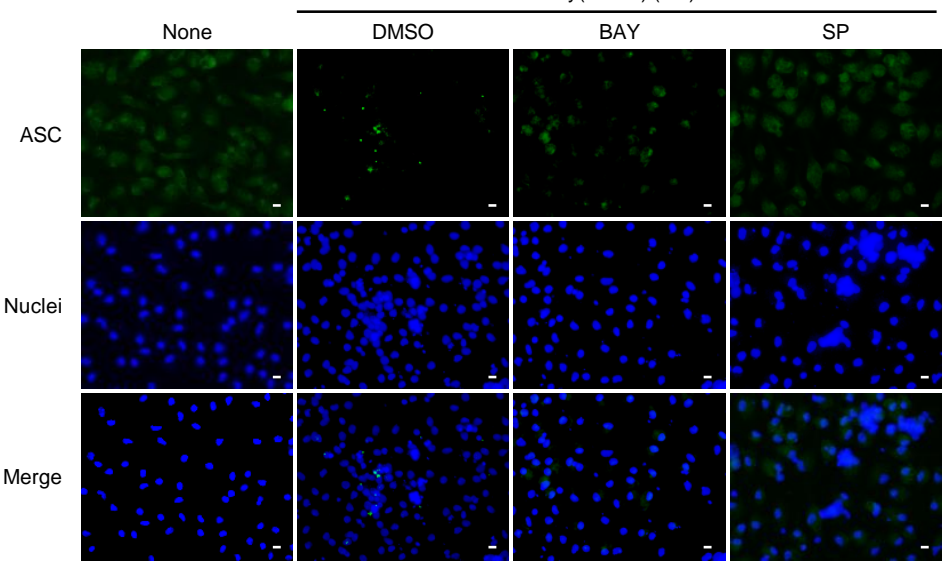

d

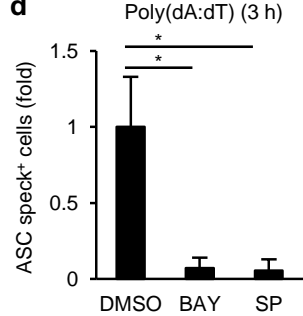

f

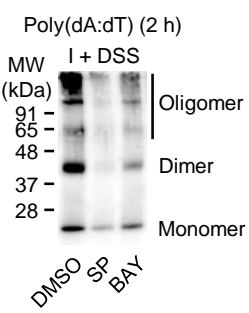

e

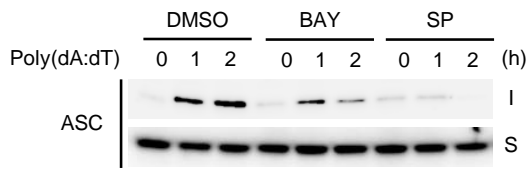

Supplementary Figure 5 Requirement of Syk and Jnk for ASC speck formation induced by poly(dA:dT). (a-d) ASC staining or (e,f) immunoblot analysis of ASC in peritoneal macrophages primed with LPS for $4 \mathrm{~h}$, followed by stimulation with nigericin for $90 \mathrm{~min}(\mathbf{a}, \mathbf{b})$, or unprimed macrophages stimulated with poly(dA:dT) for the indicated times (c-f). The kinase inhibitors were added to the cultures $1 \mathrm{~h}$ before stimulation (c-f). ASC is shown in green, nuclei in blue (a-c). The number of ASC speck-positive cell was counted and normalized to that of dimethyl sulfoxide (d). Triton-soluble (S) and Triton-insoluble (I) fractions (right margin; e) or Tritoninsoluble fractions treated with DSS (I + DSS; f). Data are shown as the means \pm s.d. of triplicate samples of one experiment. Data shown in c-f are representative of three independent experiments and those in a,b are representative of two independent experiments. Data were analyzed by Kruskal-Wallis test with Dunn's multiple comparison test $(\mathbf{d})$. Scale bar, $10 \mathrm{~mm} .{ }^{*} P<0.05$. 


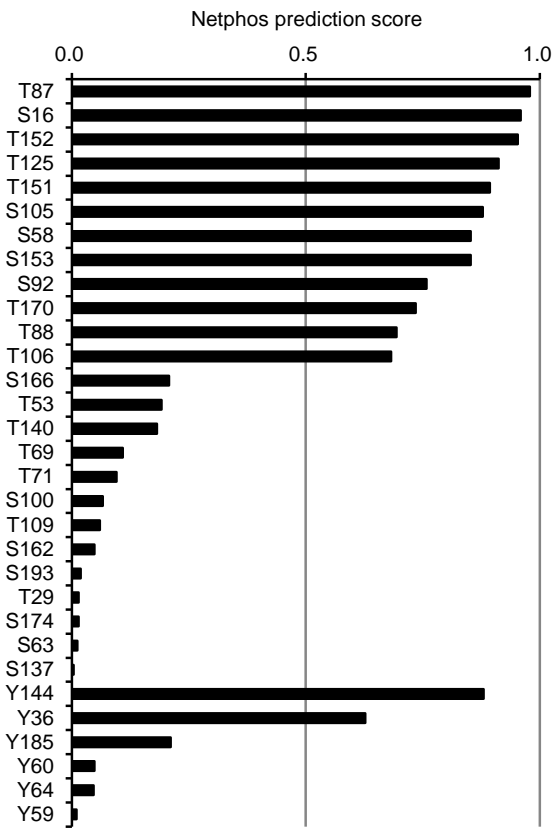

Supplementary Figure 6 Prediction of phosphorylation sites in mouse ASC. Possible phosphorylation sites in the amino acid sequence of mouse ASC were predicted by using the online program NetPhos 2.0. The threshold is 0.5 . 
a
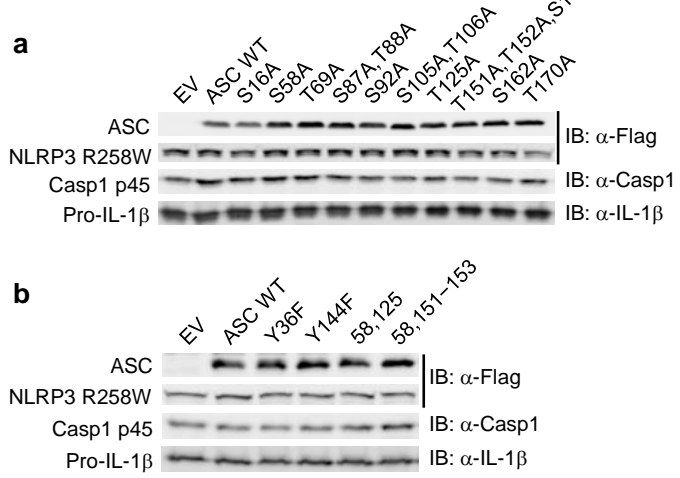

Supplementary Figure 7 Reconstitution of inflammasome system in HEK293 cells. (a, b) Immunoblot analysis of inflammasome molecules in reconstituted HEK293 cells transfected as described in Fig. 5a,b. 


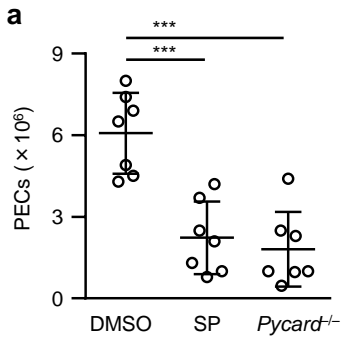

b
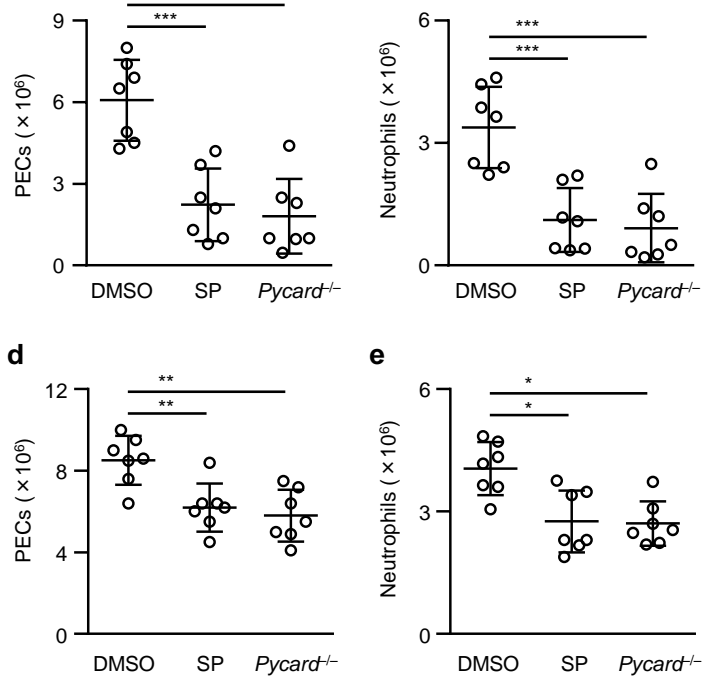

g

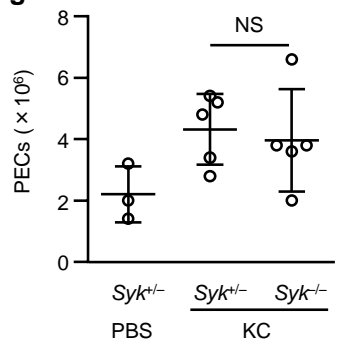

j

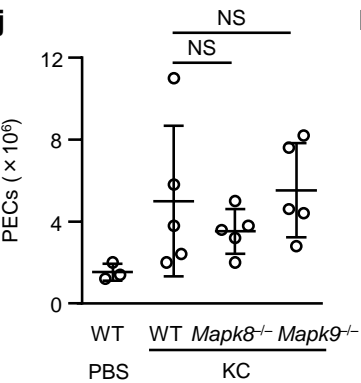

e

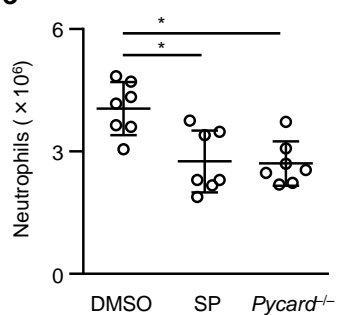

h

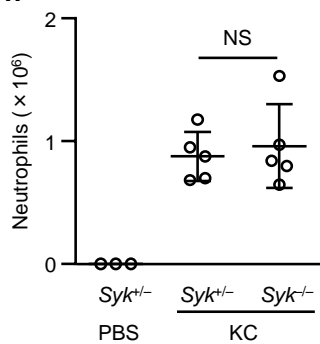

k

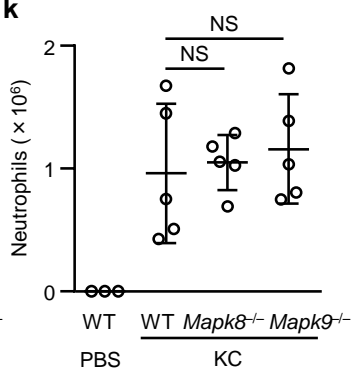

C

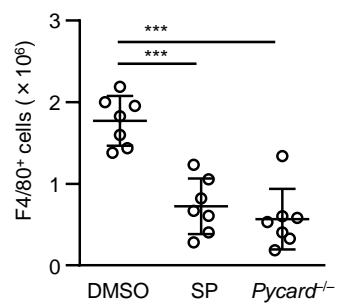

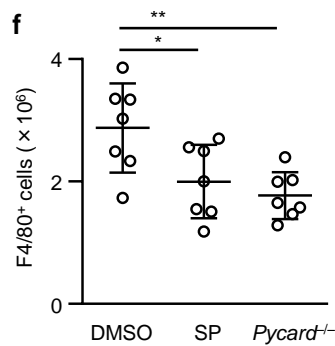

i

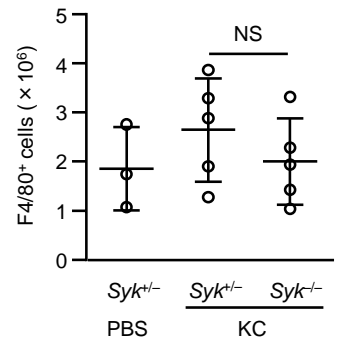

1

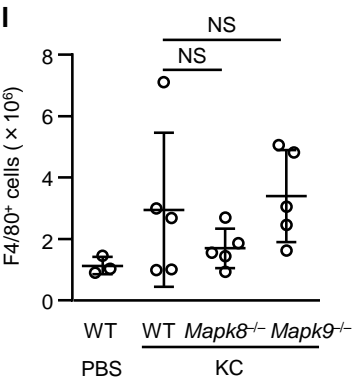

Supplementary Figure 8 Involvement of ASC and Jnk in inflammatory responses to MSU and Alum in vivo. (a-f) Infiltration of inflammatory cells in the peritoneal cavity induced by intraperitoneal injection of MSU (a-c) or alum (d-f) at $6 \mathrm{~h}$ after injection. Two hours before and $30 \mathrm{~min}$ later administration of the irritants, the mice were intraperitoneally treated with Jnk inhibitor. (g-l) Infiltration of inflammatory cells in the peritoneal cavity induced by intraperitoneal injection of $\mathrm{KC}$ or PBS at $1.5 \mathrm{~h}$ after injection. Absolute numbers of PECs (a,d,g,j), Gr- $1^{+} \mathrm{F} 4 / 80^{-}$ neutrophils $(\mathbf{b}, \mathbf{e}, \mathbf{h}, \mathbf{k})$, and F4/80+ monocytes and macrophages $(\mathbf{c}, \mathbf{f}, \mathbf{i}, \mathbf{l})$ in the peritoneum were then determined. Data are shown as dots, and the bars indicate the means \pm s.d. $(\mathrm{n}=7$ for $\mathbf{a}-\mathbf{f} ; \mathrm{n}=5$ for $\mathbf{g}-\mathbf{l} ; \mathrm{n}=3$ for PBS control in $\mathbf{g}-\mathbf{l})$. Data were analyzed by one-way ANOVA with Bonferroni (a-d,f) or Tukey-Kramer (g-l) multiple comparison test, or Kruskal-Wallis test with Dunn's multiple comparison test (e). NS, no significant difference. ${ }^{*} P<0.05,{ }^{* *} P<$ 0.01 and $^{* * * *} P<0.001$ 
Supplementary Table 1 List of kinase inhibitors

\begin{tabular}{lllr} 
Inhibitor & Abbreviation & Target & Final conc. \\
\hline R406 & R406 & Syk & $1 \mu \mathrm{M}$ \\
BAY 61-3606 & BAY & Syk & $10 \mu \mathrm{M}$ \\
Syk inhibitor I & SI & Syk & $1 \mu \mathrm{M}$ \\
PP2 & PP2 & Src & $5 \mu \mathrm{M}$ \\
SP600125 & SP & JNK & $40 \mu \mathrm{M}$ \\
TAT-TI-JIP $_{153-163}$ & TAT & JNK & $40 \mu \mathrm{M}$ \\
SB203580 & SB & p38 & $10 \mu \mathrm{M}$ \\
FR180204 & FR & Erk & $10 \mu \mathrm{M}$ \\
Wortmannin & WO & PI3K & $10 \mathrm{nM}$ \\
\hline
\end{tabular}


Supplementary Table 2 Prediction of kinase-specific phosphorylation sites in ASC from different species

\begin{tabular}{llllll} 
Tested kinase & Target protein & Code & Position & Peptide & Score \\
\hline Syk family & Mouse ASC & $\mathrm{Y}$ & 144 & SVLTEGQYQAVRAET & 1.092 \\
JNK family & Mouse ASC & $\mathrm{T}$ & 29 & KFKMKLLTVQLREGY & 1.312 \\
JNK family & Mouse ASC & $\mathrm{T}$ & 87 & ELAEQLQTTKEESGA & 1.625 \\
JNK family & Mouse ASC & $\mathrm{T}$ & 88 & LAEQLQTTKEESGAV & 1.479 \\
JNK family & Mouse ASC & $\mathrm{S}$ & 100 & GAVAAAASVPAQSTA & 1.333 \\
JNK family & Mouse ASC & $\mathrm{S}$ & 105 & AASVPAQSTARTGHF & 1.688 \\
JNK family & Mouse ASC & $\mathrm{S}$ & 153 & AVRAETTSQDKMRKL & 1.458 \\
JNK family & Mouse ASC & $\mathrm{S}$ & 193 & LVMDLEQS******* & 1.438 \\
Syk family & Human ASC & $\mathrm{Y}$ & 146 & KVLTDEQYQAVRAEP & 1.723 \\
JNK family & Human ASC & $\mathrm{Y}$ & 187 & ALRESQSYLVEDLERY & 1.308 \\
JNK family & Human ASC & $\mathrm{S}$ & 106 & GIQAPPQSAAKPGLHA & 2.500 \\
JNK family & Human ASC & $\mathrm{T}$ & 154 & QAVRAEPTNPSKMRK & 1.333 \\
JNK family & Human ASC & $\mathrm{T}$ & 166 & MRKLFSFTPAWNWTC & 2.146 \\
JNK family & Human ASC & $\mathrm{S}$ & 195 & LVEDLERS******* & 1.542 \\
Syk family & Zebrafish ASC & $\mathrm{Y}$ & 152 & KVITNEDYCTIRNKE & 1.892 \\
JNK family & Zebrafish ASC & $\mathrm{S}$ & 40 & QEPRVTKSAIEKLKD & 1.354 \\
JNK family & Zebrafish ASC & $\mathrm{T}$ & 160 & CTIRNKETPQKKMRE & 5.104 \\
JNK family & Zebrafish ASC & $\mathrm{T}$ & 170 & KKMRELLTGPITCAG & 1.521 \\
\hline
\end{tabular}


Supplementary Table 3 List of primers

\begin{tabular}{llll} 
Primer No. & Primer used for & Direction & Sequence \\
\hline 1 & mNLRP3 cloning & $\mathrm{Fw}$ & CCTGCGGCCGCAACGAGTGTCCGTTGCAAG \\
2 & mNLRP3 cloning & $\mathrm{Rv}$ & CCTGGTACCCTACCAGGAAATCTCGAAGACTA \\
3 & mSyk cloning & $\mathrm{Fw}$ & AGCTTGCGGCCGCGGGAAGTGCTGTGGACAGCGCC \\
4 & mSyk cloning & $\mathrm{Rv}$ & CTAGAGTCGACTTAGTTAACCACGTCGTAGTAG \\
5 & mJNK1 cloning & $\mathrm{Fw}$ & CAACTATCGATGAGCAGAAGCAAACGTGACAAC \\
6 & mJNK1 cloning & $\mathrm{Rv}$ & CGCACGGATCCTCATTGCTGCACCTGTGCTAAAGG \\
7 & mJNK2 cloning & $\mathrm{Fw}$ & CAACTATCGATGAGTGACAGTAAAAGCGATGG \\
8 & mJNK2 cloning & $\mathrm{Rv}$ & CGCACGGATCCTCACCGGCAGCCTTCCAGGGGTCC \\
9 & NLRP3-R258W mutant & $\mathrm{Fw}$ & CCACTGCTGGGAGGTGAGCCTCAG \\
10 & NLRP3-R258W mutant & $\mathrm{Rv}$ & TCACCTCCCAGCAGTGGATAAAGAA \\
11 & ASC-S16A mutant & $\mathrm{Fw}$ & AACTTGGCCGGGGATGAACTCAAAAAG \\
12 & ASC-S16A mutant & $\mathrm{Rv}$ & ATCCCCGGCCAAGTTTTCAAGAGC \\
13 & ASC-S58A mutant & $\mathrm{Fw}$ & CTTGTCGCCTACTATCTGGAGTCGTATG \\
14 & ASC-S58A mutant & $\mathrm{Rv}$ & ATAGTAGGCGACAAGTTTGTCAGTGAG \\
15 & ASC-T69A mutant & $\mathrm{Fw}$ & GAGCTCGCCATGACTGTGCTTAGAG \\
16 & ASC-T69A mutant & $\mathrm{Rv}$ & AGTCATGGCGAGCTCCAAGCCATAC \\
17 & ASC-S87A/T88A mutant & $\mathrm{Fw}$ & CTGCAAGCCGCTAAAGAAGAGTCTGGA \\
18 & ASC-S87A/T88A mutant & $\mathrm{Rv}$ & CTTCTTTAGCGGCTTGCAGCTGCTCAGC \\
\hline
\end{tabular}




\begin{tabular}{llll} 
Primer No. & Primer used for & Direction & Sequence \\
\hline 19 & ASC-S92A mutant & $\mathrm{Fw}$ & GAAGAGGCCGGAGCTGTGGCAGCTG \\
20 & ASC-S92A mutant & $\mathrm{Rv}$ & CAGCTCCGGCCTCTTCTTTAGTCGT \\
21 & ASC-S105A/T106A mutant & $\mathrm{Fw}$ & CTCAGGCCGCAGCCAGAACAGGACAC \\
22 & ASC-S105A/T106A mutant & $\mathrm{Rv}$ & CTGGCTGCGGCCTGAGCAGGGACACTG \\
23 & ASC-T125A mutant & $\mathrm{Fw}$ & AGGGTCGCAGAAGTGGACGGAGTGCTG \\
24 & ASC-T125A mutant & $\mathrm{Rv}$ & CACTTCTGCGACCCTGGCAATGAGTGC \\
25 & ASC-T151A/T152A/S153A mutant & $\mathrm{Fw}$ & AGAGGCCGCCGCCCAAGACAAGATGAGGAAG \\
26 & ASC-T151A/T152A/S153A mutant & $\mathrm{Rv}$ & CTTGGGCGGCGGCCTCTGCACGAACTGCCTG \\
27 & ASC-S162A mutant & $\mathrm{Fw}$ & AACTTGGCCGGGGATGAACTCAAAAAG \\
28 & ASC-S162A mutant & $\mathrm{Rv}$ & ATCCCCGGCCAAGTTTCAAGAGC \\
29 & ASC-T170A mutant & $\mathrm{Fw}$ & AACCTGGCCTGCAAGGACTCCCTC \\
30 & ASC-T170A mutant & $\mathrm{Rv}$ & CTTGCAGGCCAGGTTCCAGGATGG \\
31 & ASC-Y36F mutant & $\mathrm{Fw}$ & GAAGGCTTTGGGCGCATCCCACGC \\
32 & ASC-Y36F mutant & $\mathrm{Rv}$ & GCGCCCAAAGCCTTCTCGCAGTTG \\
33 & ASC-Y144F mutant & $\mathrm{Fw}$ & GGACAGTTCCAGGCAGTTCGTGCA \\
34 & ASC-Y144F mutant & $\mathrm{Rv}$ & TGCCTGGAACTGTCCTTCAGTCAG \\
35 & Deletion of FLAG-tag & $\mathrm{Fw}$ & CTACCATGGCGGCCGCGAATTCATCG \\
36 & Deletion of FLAG-tag & $\mathrm{Rv}$ & GCGGCCGCCATGGTAGATCAATTCTGA \\
\hline
\end{tabular}

